\title{
Markovian Solutions to Discontinuous ODEs
}

\author{
Alberto Bressan ${ }^{(1)}$, Marco Mazzola ${ }^{(2)}$, and Khai T. Nguyen ${ }^{(3)}$ \\ (1) Department of Mathematics, Penn State University, \\ (2) IMJ-PRG, CNRS, Sorbonne Université, \\ (3) Department of Mathematics, North Carolina State University. \\ e-mails: axb62@psu.edu, marco.mazzola@imj-prg.fr, khai@math.ncsu.edu
}

September 15, 2020

\begin{abstract}
Given a possibly discontinuous, bounded function $f: \mathbb{R} \mapsto \mathbb{R}$, we consider the set of generalized flows, obtained by assigning a probability measure on the set of Carathéodory solutions to the ODE $\dot{x}=f(x)$. The paper provides a complete characterization of all such flows which have a Markov property in time. This is achieved in terms of (i) a positive, atomless measure supported on the set $f^{-1}(0)$ where $f$ vanishes, (ii) a countable number of Poisson random variables, determining the waiting times at points in $f^{-1}(0)$, and (iii) a countable set of numbers $\theta_{k} \in[0,1]$, describing the probability of moving up or down, at isolated points where two distinct trajectories can originate.
\end{abstract}

\section{Introduction}

Consider a scalar ODE with possibly discontinuous right hand side:

$$
\dot{x}=f(x) .
$$

Given an initial condition

$$
x(0)=x_{0},
$$

we recall that $t \mapsto x(t)$ is a Carathéodory solution of the Cauchy problem (1.1)-(1.2) if

$$
x(t)=x_{0}+\int_{0}^{t} f(x(s)) d s \quad \text { for all } t \geq 0 .
$$

When the function $f$ is not Lipschitz continuous, it is well known that this Cauchy problem can admit multiple solutions. Because of this non-uniqueness, in [16] it was proposed to study "generalized flows", described by a probability measure on the set of all Carathéodory solutions. See also [18] for a related approach. In this direction, the main goal of the present 
paper is to describe all stochastic flows compatible with the ODE, which have the Markov property. Toward this goal, in the first part of the paper we describe all possible deterministic semigroups:

Definition 1.1 By a deterministic semigroup compatible with the ODE (1.1) we mean a map $S: \mathbb{R} \times \mathbb{R}_{+} \mapsto \mathbb{R}$, with the properties

(i) $S_{t}\left(S_{s}\left(x_{0}\right)\right)=S_{t+s}\left(x_{0}\right), S_{0}\left(x_{0}\right)=x_{0}$.

(ii) For each $x_{0} \in \mathbb{R}$, the map $t \mapsto S_{t} x_{0}$ is a solution to the Cauchy problem (1.1)-(1.2).

Notice that here we do not require any continuity w.r.t. the initial point $x_{0}$. Throughout the following, as basic assumptions we consider:

(A1) The function $f: \mathbb{R} \mapsto \mathbb{R}$ is bounded and regulated, i.e. it admits left and right limits $f(x-), f(x+)$ at every point $x$.

(A2) If $y$ is a point where either $f(y-) \cdot f(y+)=0$ or else $f(y-)>0>f(y+)$, then $f(y)=0$.

Remark 1.1 The assumption (A2) corresponds to the "no-jamming" condition used in [4]. It is needed to rule out examples such as

$$
\dot{x}=f(x)=\left\{\begin{array}{rl}
1 & \text { if } x<0, \\
-1 & \text { if } x>0, \\
a & \text { if } x=0,
\end{array} \quad x(0)=0,\right.
$$

which has no Carathéodory solution if $a \neq 0$.

Remark 1.2 It is well known that every regulated function has at most countably many points of discontinuity. Namely, the set

$$
D_{f}=\{x \in \mathbb{R} ; f(x-) \neq f(x+) \text { or } f(x-)=f(x+) \neq f(x)\}
$$

is at most countable. We observe that, under the assumptions (A1)-(A2), the set of zeroes

$$
f^{-1}(0)=\{x \in \mathbb{R} ; f(x)=0\}
$$

is closed. Indeed, assume that $f\left(x_{n}\right)=0$ for a strictly increasing sequence $x_{n} \rightarrow \bar{x}$. By (A1) this implies that the left limit exists and satisfies $f(\bar{x}-)=0$. Hence (A2) yields $f(\bar{x})=0$. Similarly, if $x_{n}$ decreases to $\bar{x}$, then $f(\bar{x}+)=0$ and hence (A2) again implies $f(\bar{x})=0$.

In Section 3, we will show that, in order to uniquely determine a deterministic semigroup compatible with (1.1), three additional ingredients are needed:

(Q1) A continuum (i.e., atomless) positive measure $\mu$ supported on the set of zeroes (1.5) of $f$. Strictly increasing trajectories $t \mapsto x(t)=S_{t}\left(x_{0}\right)$ of the semigroup are then defined by the identity

$$
t=\int_{x_{0}}^{x(t)} \frac{d y}{f(y)}+\mu\left(\left[x_{0}, x(t)\right]\right)
$$

while a similar formula holds for decreasing ones. Notice that, if $f^{-1}(0)$ is countable, then necessarily $\mu=0$. 
(Q2) A countable set of points $\mathcal{S} \subseteq f^{-1}(0)$, where the dynamics is forced to stop. Among the (possibly many) solutions of (1.1) starting from $x_{0} \in \mathcal{S}$, we thus choose $S_{t}\left(x_{0}\right)=x_{0}$.

(Q3) A map $\Phi: \Omega^{*} \mapsto\{-1,1\}$, defined on a set $\Omega^{*} \subset \mathbb{R}$ of isolated points from where both an increasing and a decreasing solution of (1.1) can originate. For $x_{0} \in \Omega^{*}$, setting $\Phi\left(x_{0}\right)=1$ selects the increasing solution, while $\Phi\left(x_{0}\right)=-1$ selects the decreasing one.

In the second part of the paper we study Markov processes, whose sample paths are solutions to (1.1). Given a deterministic semigroup compatible with (1.1), in Section 4 we show that a Markov semigroup can be obtained by adding two more ingredients to the list (Q1)-(Q3). Namely:

(Q4) A countable set $\mathcal{S}^{*} \subset f^{-1}(0)$ and a map $\left.\Lambda: \mathcal{S}^{*} \mapsto\right] 0,+\infty[$, describing the random waiting time of a trajectory which reaches a point $y_{j} \in \mathcal{S}^{*}$. More precisely, we assume that a solution initially at $y_{j} \in \mathcal{S}^{*}$ remains at $y_{j}$ for a random waiting time $Y_{j} \geq 0$, then starts moving. All these random times will be mutually independent, with Poisson distribution:

$$
\text { Prob. }\left\{Y_{j}>s\right\}=e^{-\lambda_{j} s} \quad \text { with } \quad \lambda_{j}=\Lambda\left(y_{j}\right)
$$

(Q5) A map $\Theta: \Omega^{*} \mapsto[0,1]$, defined on the countable set $\Omega^{*} \subset \mathbb{R}$ of points from which both an increasing and a decreasing solution of (1.1) can originate. For $x_{k} \in \Omega^{*}$, the value $\theta_{k}=\Theta\left(x_{k}\right)$ gives the probability that, when the solution starting from $x_{k}$ begins to move, it will be increasing. Of course, $1-\theta_{k}$ is then the probability that the solution will be decreasing.

Conversely, in Section 5 we prove that every Markov semigroup whose sample paths are solutions to (1.1) can be obtained by first constructing a deterministic semigroup $S$ as in (Q1)(Q3), and then adding the random waiting times $Y_{j}$ in (Q4) and the upward vs. downward probabilities $\theta_{k}$ in (Q5).

Example 1.1 Given $0<\alpha<1$, consider the ODE

$$
\dot{x}=\frac{|x|^{\alpha}}{1-\alpha} .
$$

This provides a classical example of an ODE with continuous right hand side, with multiple solutions starting at the origin. This ODE is compatible with two deterministic semigroups $S, \widetilde{S}: \mathbb{R} \times \mathbb{R}_{+} \mapsto \mathbb{R}$. The first one satisfies $S_{t}(0)=0$ for all $t \geq 0$, while the second one satisfies $\widetilde{S}_{t}(0)=t^{\frac{1}{1-\alpha}}$. In addition, there is a one-parameter family of Markovian flows. Indeed, for each $0<\lambda<\infty$, one can consider the random time $T$ with Poisson distribution

$$
\text { Prob. }\{T>s\}=e^{-\lambda s} \text {. }
$$

A random solution starting at $x=0$ remains at the origin up to time $T$, then it is strictly increasing for $t>T$, namely $x(t)=(t-T)^{\frac{1}{1-\alpha}}$. Notice that, by taking the limits $\lambda \rightarrow 0$ or $\lambda \rightarrow+\infty$, we recover the deterministic semigroups $S$ and $\widetilde{S}$, respectively. 
The remainder of the paper is organized as follows. In Section 2 we briefly review some basic properties of scalar ODEs with discontinuous right hand side. For the main results on existence and uniqueness of solutions we refer to [4]. These are based on the elementary solution formula

$$
\int_{x_{0}}^{x(t)} \frac{1}{f(y)} d y=t
$$

valid on intervals where $f$ has a.e. the same sign, and $1 / f$ is integrable.

Section 3 is concerned with deterministic semigroups. Theorem 3.1 describes the most general semigroup $S: \mathbb{R} \times \mathbb{R}_{+} \mapsto \mathbb{R}$ compatible with the ODE (1.1). In Section 4 we construct a family of Markov semigroups whose sample paths are solutions to (1.1). Each of these semigroups is determined by the additional data introduced at (Q1)-(Q5). Finally, Theorem 5.1 in Section 5 shows that every Markov semigroup compatible with (1.1) is one of the above family.

In the companion paper [7], the authors show that every deterministic semigroup compatible with (1.1) can be obtained as the pointwise limit of the flows generated by a sequence of ODEs $\dot{x}=f_{n}(x)$ with smooth right hand sides. Moreover, every Markovian semigroup can be obtained as limit of a sequence of diffusion processes with smooth drifts and with diffusion coefficients approaching zero.

Ordinary differential equations with discontinuous right hand side have been studied for their own interest $[4,5,6,17,20]$, as well as for their several applications to feedback control $[3,9,19,21]$. They also play a key role in the theory of transport equations $[1,2,10,15]$, and in the analysis of solutions to hyperbolic conservation laws by the method of characteristics $[8,11,12,13]$. For a survey of numerical methods we refer to [14].

\section{Review of scalar discontinuous ODEs}

As a preliminary, we collect here some basic results on the existence and properties of solutions to a Cauchy problem with possibly discontinuous right hand side. In the following theorem, the first two statements can already be found in [4], where a more general setting was considered.

However, the closure of the solution set strongly relies on the assumption (A1) that the function $f$ is regulated.

Theorem 2.1 Consider the Cauchy problem (1.1)-(1.2), assuming that (A1)-(A2) hold. Then

(i) For every $x_{0} \in \mathbb{R}$ there exists at least one Carathéodory solution, defined for all times $t \geq 0$.

(ii) Every solution is monotone (either increasing or decreasing).

(iii) The set of all solutions is nonempty and closed w.r.t. the topology of uniform convergence on bounded sets. 
Proof. 1. We begin by proving that (1.1)-(1.2) admits a Carathéodory solution. By the assumption (A1) there exists a constant $M>0$ such that

$$
|f(x)| \leq M \quad \text { for all } x \in \mathbb{R} .
$$

Let any $x_{0} \in \mathbb{R}$ be given. If $f\left(x_{0}\right)=0$ then trivially $x(t) \equiv x_{0}$ is a solution of (1.1). On the other hand, if $f\left(x_{0}\right) \neq 0$, then by the assumption (A2) we must have

$$
\text { either } f\left(x_{0}+\right)>0 \text { or } f\left(x_{0}-\right)<0 \text {. }
$$

To fix the ideas, assume $f\left(x_{0}+\right)>0$, the other case being entirely similar. We can then find $\delta, h>0$ such that

$$
\left.h \leq f(x) \leq M \quad \text { for all } x \in] x_{0}, x_{0}+\delta\right]
$$

The map

$$
x \mapsto t(x) \doteq \int_{x_{0}}^{x} \frac{d y}{f(y)}
$$

is thus strictly increasing in the interval $\left[x_{0}, x_{0}+\delta\right]$. Indeed, for the above inequality, for all $x_{0} \leq x_{1}<x_{2} \leq x_{0}+\delta$ it holds

$$
\frac{x_{2}-x_{1}}{M} \leq t\left(x_{2}\right)-t\left(x_{1}\right)=\int_{x_{1}}^{x_{2}} \frac{d y}{f(y)} \leq \frac{x_{2}-x_{1}}{h} .
$$

This implies that the inverse map $t \mapsto x(t)$ is strictly increasing and Lipschitz continuous. Hence it is absolutely continuous, and satisfies

$$
\dot{x}(t)=\left(\frac{d}{d x} t(x)\right)^{-1}=f(x(t)) \text { for a.e. } t \in\left[t\left(x_{0}\right), t\left(x_{0}+\delta\right)\right] .
$$

Thus, $x(t)$ is a solution of (1.1), defined on the interval $\left[t\left(x_{0}\right), t\left(x_{0}+\delta\right)\right]$.

We claim that this solution can be extended for all $t \in[0,+\infty[$. Indeed, if $[0, T$ [ is a maximal domain where the solution can be constructed, since $f$ is bounded by $M$, the limit $x(T) \doteq$ $\lim _{t \rightarrow T-} x(t)$ exists. By the previous arguments, the solution can then be extended to $[0, T+\delta]$ for some $\delta>0$, contradicting the maximality assumption.

2. To prove that every solution is monotone, let $t \mapsto x(t)$ be a Carathéodory solution of (1.1). Assume, on the contrary, that $x(\cdot)$ is neither monotone increasing nor decreasing. Without loss of generality we can assume that

$$
x\left(t_{1}\right)=x\left(t_{2}\right)<x(\bar{t}) \quad \text { for some } t_{1}<\bar{t}<t_{2} .
$$

Since $x(\cdot)$ is absolutely continuous, there exists $\left.\tau_{1} \in\right] t_{1}, t_{2}[$ such that $x(\cdot)$ is differentiable at $\tau_{1}$ and

$$
\dot{x}\left(\tau_{1}\right)>0, \quad x\left(t_{1}\right)<x\left(\tau_{1}\right)<x(\bar{t}) .
$$

Therefore there exists $\delta_{0}>0$ such that

$$
\left.x\left(\tau_{1}\right)<x\left(\tau_{1}+s\right) \quad \text { for all } s \in\right] 0, \delta_{0}[.
$$


Since $f$ has a right limit at $x\left(\tau_{1}\right)$, we deduce

$$
0<\dot{x}\left(\tau_{1}\right)=\lim _{h \rightarrow 0+} \frac{1}{h} \int_{0}^{h} f\left(x\left(\tau_{1}+s\right)\right) d s=f\left(x\left(\tau_{1}\right)+\right) .
$$

On the other hand, since $x\left(t_{2}\right)<x\left(\tau_{1}\right)<x(\bar{t})$, we consider the time

$$
\tau_{2} \doteq \min \left\{t \geq \bar{t} \quad: \quad x(t)=x\left(\tau_{1}\right)\right\} .
$$

The above definition implies $x(t)>x\left(\tau_{1}\right)$ for all $t \in\left[\bar{t}, \tau_{2}[\right.$ while

$$
x\left(\tau_{2}\right)=\lim _{t \rightarrow \tau_{2}-} x(t)=x\left(\tau_{1}\right) .
$$

As a consequence, we have

$$
f\left(x\left(\tau_{1}\right)+\right)=\lim _{h \rightarrow 0+} \frac{1}{h} \int_{-h}^{0} f\left(x\left(\tau_{2}+s\right)\right) d s=\lim _{h \rightarrow 0+} \frac{x\left(\tau_{2}\right)-x\left(\tau_{2}-h\right)}{h} \leq 0,
$$

reaching a contradiction with (2.2).

3. To prove (iii), consider a sequence of solutions $x_{n}(\cdot)$ of $(1.1)$, converging to a function $\bar{x}(\cdot)$ uniformly on bounded sets. We claim that $\bar{x}$ is also a Carathéodory solution. To fix the ideas, assume that all solutions $x_{n}$ are monotone increasing. Hence $\bar{x}$ is monotone increasing as well. We need to show that

$$
\bar{x}(b)=\bar{x}(a)+\int_{a}^{b} f(\bar{x}(s)) d s
$$

for any interval $[a, b]$. Toward this goal, consider the set of times

$$
\begin{aligned}
I_{0}=\{t \in[a, b] ; & \text { there exists } \varepsilon>0 \text { such that } \\
\bar{x}(\cdot) & \text { is constant either on }[t, t+\varepsilon] \text { or on }[t-\varepsilon, t]\} .
\end{aligned}
$$

We claim that

$$
\bar{x}(t) \in f^{-1}(\{0\}) \quad \text { for all } t \in I_{0} .
$$

Indeed, by the definition of $I_{0}$, for any $t_{0} \in I_{0}$ there exist $t_{1}<t_{2}$ with $t_{0} \in\left[t_{1}, t_{2}\right]$ such that

$$
\bar{x}(t)=\bar{x}\left(t_{0}\right) \quad \text { for all } t \in\left[t_{1}, t_{2}\right] .
$$

Hence,

$$
\lim _{n \rightarrow \infty} \int_{t_{1}}^{t_{2}} f\left(x_{n}(t)\right) d t=\lim _{n \rightarrow \infty}\left[x_{n}\left(t_{2}\right)-x_{n}\left(t_{1}\right)\right]=0 .
$$

Since $x_{n}$ is monotone increasing, we have $f\left(x_{n}(s)\right)=x_{n}^{\prime}(s) \geq 0$ for a.e. $s$. This implies

$$
\lim _{n \rightarrow \infty}\left\|f\left(x_{n}\right)\right\|_{\mathbf{L}^{1}\left(\left[t_{1}, t_{2}\right]\right)}=0 .
$$

By possibly taking a subsequence, this yields the pointwise convergence $f\left(x_{n}(t)\right) \rightarrow 0$ for a.e. $t \in\left[t_{1}, t_{2}\right]$.

As a consequence, one of the three following cases must hold: 
(i) $f\left(x\left(t_{0}\right)\right)=0$,

(ii) $f\left(x\left(t_{0}\right)-\right)=0$,

(iii) $f\left(x\left(t_{0}\right)+\right)=0$.

By the assumption (A2), both (ii) and (iii) imply (i), proving our claim (2.4). In particular, this implies

$$
\lim _{n \rightarrow \infty} f\left(x_{n}(t)\right)=f(\bar{x}(t))=0 \quad \text { for a.e. } t \in I_{0} .
$$

Next, from the definition of $I_{0}$ it follows that the function $\bar{x}(\cdot)$ is one-to-one (strictly increasing) on $[a, b] \backslash I_{0}$. In particular, this implies that the set

$$
\left\{t \in[a, b] \backslash I_{0} ; \quad \bar{x}(t) \in D_{f}\right\}
$$

is at most countable. Since $f$ is continuous outside $D_{f}$ and $x_{n}(t)$ converges to $\bar{x}(t)$ for every $t \in[a, b]$, we have

$$
\lim _{n \rightarrow \infty} f\left(x_{n}(t)\right)=f(\bar{x}(t)) \quad \text { for a.e. } t \in[a, b] \backslash I_{0} .
$$

By (2.5)-(2.6), the dominated convergence theorem yields

$$
\lim _{n \rightarrow \infty} \int_{a}^{b} f\left(x_{n}(t)\right) d s=\int_{a}^{b} f(\bar{x}(t)) d t .
$$

This proves (2.3).

Example 2.1 To appreciate the importance of the assumption (A1), consider the following Cauchy problem, proposed in [4].

$$
\dot{x}=f(x)=\left\{\begin{array}{ll}
0 & \text { if } \quad x=1, \frac{1}{2}, \frac{1}{3}, \ldots \\
1 & \text { otherwise, }
\end{array} \quad x(0)=0 .\right.
$$

Then for every $n \geq 1$ the function

$$
x_{n}(t)=\min \left\{\frac{1}{n}, t\right\}
$$

is a Carathéodory solution, but the uniform limit $x(t)=\lim _{n \rightarrow \infty} x_{n}(t)=0$ does not satisfy (2.7). Notice that the function $f$ in (2.7) is not regulated.

\section{Semigroups compatible with the ODE}

In general, the Cauchy problem for the discontinuous ODE (1.1)-(1.2) admits several different solutions. Our present goal is to study all possible semigroups $S: \mathbb{R} \times \mathbb{R}_{+} \mapsto \mathbb{R}$ compatible with the ODE (1.1), in the sense of Definition 1.1.

We first introduce a few definitions. For any function $f$, denote

$$
f^{+}(x) \doteq \max \{f(x), 0\}, \quad f^{-}(x)=\min \{f(x), 0\} .
$$


If $f$ is regulated, so are $f^{+}, f^{-}$. Moreover, we define the sets

$$
\begin{aligned}
& \mathcal{R}^{-} \doteq\left\{x \in \mathbb{R} ; \quad \int_{x-\varepsilon}^{x} \frac{1}{f^{-}(y)} d y>-\infty \text { for some } \varepsilon>0\right\}, \\
& \mathcal{R}^{+} \doteq\left\{x \in \mathbb{R} ; \quad \int_{x}^{x+\varepsilon} \frac{1}{f^{+}(y)} d y<+\infty \text { for some } \varepsilon>0\right\} .
\end{aligned}
$$

Notice that $\bar{x} \in \mathcal{R}^{-}$if there exists a strictly decreasing Carathéodory solution to (1.1), say $x:[0, \tau] \mapsto \mathbb{R}$, with $\tau>0$, such that $x(0)=\bar{x}$. Similarly, $\bar{x} \in \mathcal{R}^{+}$if there exists a strictly increasing Carathéodory solution to (1.1) which starts at $\bar{x}$.

From the above definitions, it immediately follows that $\mathcal{R}^{-}$is open to the left and $\mathcal{R}^{+}$is open to the right. Namely

$$
\begin{aligned}
& \left.\left.x \in \mathcal{R}^{-} \quad \Longrightarrow \quad\right] x-\delta, x\right] \subset \mathcal{R}^{-} \quad \text { for some } \delta>0, \\
& x \in \mathcal{R}^{+} \quad \Longrightarrow \quad\left[x, x+\delta\left[\subset \mathcal{R}^{+} \quad \text { for some } \delta>0 .\right.\right.
\end{aligned}
$$

For future use, we also define the sets

$$
\Omega^{*} \doteq \mathcal{R}^{-} \cap \mathcal{R}^{+}, \quad \Omega_{0} \doteq \mathbb{R} \backslash\left(\mathcal{R}^{-} \cup \mathcal{R}^{+}\right) .
$$

Notice that $\Omega^{*}$ is the set of points from which two distinct solutions of (1.1) can initiate: one strictly decreasing, and one strictly increasing. On the other hand, $\Omega_{0}$ is a set of points from which neither an increasing nor a decreasing solution can initiate. If $x_{0} \in \Omega_{0}$, the unique solution to the Cauchy problem (1.1)-(1.2) is the constant one: $x(t)=x_{0}$ for all $t \geq 0$.

Remark 3.1 By (A2), the set of zeroes $f^{-1}(0)$ is closed. Moreover, the assumptions (A1)(A2) imply the inclusion $\Omega_{0} \subseteq f^{-1}(0)$. We also observe that the set $\Omega^{*}$ consists of isolated points. Therefore, there can be at most countably many such points.

Remark 3.2 In general, the set

$$
f^{-1}(0) \cap\left(\mathcal{R}^{+} \cup \mathcal{R}^{-}\right)
$$

has measure zero, hence its interior is empty. However, it may well be uncountable. For example let $\mathbf{C} \subset[0,1]$ be the Cantor set, which we write in the form

$$
\mathbf{C}=[0,1] \backslash \bigcup_{n=0}^{\infty} \bigcup_{k=1}^{2^{n}} I_{k}^{n}
$$

where $\left.I_{k}^{n}=\right] a_{k}^{n}, b_{k}^{n}$ [ are disjoint open intervals with length $3^{-(n+1)}$. We define

$$
f(x)=\left\{\begin{array}{cl}
0 & \text { if } x \in \mathbf{C} \cup]-\infty, 0] \cup[1,+\infty[, \\
\min \left\{\left|x-a_{k}^{n}\right|^{1 / 3},\left|x-b_{k}^{n}\right|^{1 / 3}\right\} & \text { if } x \in I_{k}^{n} .
\end{array}\right.
$$

It is easy to check that $f$ is continuous and

$$
\int_{[0,1] \backslash \mathbf{C}} \frac{1}{f(x)} d x=\sum_{n=0}^{\infty} \sum_{k=1}^{2^{n}} \int_{I_{k}^{n}} \frac{1}{f(x)} d x=3 \sum_{n=0}^{\infty} \sum_{k=1}^{2^{n}}\left(\frac{3^{-(n+1)}}{2}\right)^{2 / 3}
$$




$$
=\frac{3^{1 / 3}}{2^{2 / 3}} \cdot \sum_{n=0}^{\infty}\left(\frac{2}{3^{2 / 3}}\right)^{n}<+\infty .
$$

This provides an example where the set $f^{-1}(0) \cap \mathcal{R}^{+}=\mathbf{C} \backslash\{1\}$ is uncountable. As a consequence, this set can support a nontrivial atomless measure $\mu$.

We now consider the ODE (1.1), together with a positive, atomless measure $\mu$ supported on $f^{-1}(0)$, a countable set $\mathcal{S} \subseteq f^{-1}(0)$, and a map $\Phi: \Omega^{*} \mapsto\{-1,1\}$, as described at (Q1)(Q3) in the Introduction. We claim that these assignments uniquely determine a semigroup $S: \mathbb{R} \times \mathbb{R}_{+} \mapsto \mathbb{R}$, compatible with the ODE (1.1).

Definition 3.1 We say that an open interval $] a, b[$ is a domain of increase if

$$
] a, b\left[\cap \mathcal{S}=\emptyset, \quad \mu([c, d])+\int_{c}^{d} \frac{d x}{f^{+}(x)}<+\infty \quad \text { for all }[c, d] \subset\right] a, b[.
$$

Similarly, we say that $] a, b[$ is a domain of decrease if

$$
] a, b\left[\cap \mathcal{S}=\emptyset, \quad \mu([c, d])-\int_{c}^{d} \frac{d x}{f^{-}(x)}<+\infty \quad \text { for all }[c, d] \subset\right] a, b[.
$$

If $] a, b[$ and $] a^{\prime}, b^{\prime}[$ are two intervals of increase having non-empty intersection, then their union $] a, b[\cup] a^{\prime}, b^{\prime}[$ is also an interval of increase. We can thus identify countably many, disjoint maximal intervals $] \alpha_{i}, \beta_{i}\left[, i \in \mathcal{I}^{+}\right.$of increase. Similarly, we can identify countably many disjoint maximal intervals $] \gamma_{i}, \delta_{i}\left[, i \in \mathcal{I}^{-}\right.$of decrease.

It remains to analyze what happens at the endpoints of these intervals.

- If $\alpha_{i} \notin \mathcal{S}$ and, for some $\varepsilon>0$

$$
\mu\left(\left[\alpha_{i}, \alpha_{i}+\varepsilon\right]\right)+\int_{\alpha_{i}}^{\alpha_{i}+\varepsilon} \frac{d x}{f^{+}(x)}<+\infty
$$

we then consider the half-open interval $I_{i}^{+} \doteq\left[\alpha_{i}, \beta_{i}\left[\right.\right.$. Otherwise, we let $I_{i}^{+}$be an open interval: $\left.I_{i}^{+} \doteq\right] \alpha_{i}, \beta_{i}[$.

- If $\delta_{i} \notin \mathcal{S}$ and, for some $\varepsilon>0$

$$
\mu\left(\left[\delta_{i}-\varepsilon, \delta_{i}\right]\right)-\int_{\delta_{i}-\varepsilon}^{\delta_{i}} \frac{d x}{f^{-}(x)}<+\infty
$$

we then consider the half-open interval $\left.\left.I_{i}^{-} \doteq\right] \gamma_{i}, \delta_{i}\right]$. Otherwise, we let $I_{i}^{-}$be an open interval: $\left.I_{i}^{-} \doteq\right] \gamma_{i}, \delta_{i}[$.

For each $i \in \mathcal{I}^{+}$, we now describe the increasing dynamics on the intervals $I_{i}^{+}$. Given $x_{0} \in I_{i}^{+}$, we consider the time

$$
\left.\left.\tau^{+}\left(x_{0}\right) \doteq \mu\left(\left[x_{0}, \beta_{i}\right]\right)+\int_{x_{0}}^{\beta_{i}} \frac{d y}{f^{+}(y)} \in\right] 0,+\infty\right] .
$$


We then set

$$
S_{t}^{+}\left(x_{0}\right) \doteq x(t)
$$

where $x(t)$ is implicitly defined by

$$
\begin{array}{cc}
\mu\left(\left[x_{0}, x(t)\right]\right)+\int_{x_{0}}^{x(t)} \frac{d y}{f^{+}(y)}=t & \text { if } t<\tau^{+}\left(x_{0}\right), \\
x(t)=\beta_{i} & \text { if } t \geq \tau^{+}\left(x_{0}\right) .
\end{array}
$$

The construction of the decreasing dynamics on the intervals $I_{i}^{-}$is entirely similar. Given $x_{0} \in I_{i}^{-}$, we consider the time

$$
\left.\left.\tau^{-}\left(x_{0}\right) \doteq \mu\left(\left[\gamma_{i}, x_{0}\right]\right)-\int_{\gamma_{i}}^{x_{0}} \frac{d y}{f^{+}(y)} \in\right] 0,+\infty\right] .
$$

We then set

$$
S_{t}^{-}\left(x_{0}\right) \doteq x(t)
$$

where $x(t)$ is implicitly defined by

$$
\begin{array}{cc}
\mu\left(\left[x(t), x_{0}\right]\right)-\int_{x(t)}^{x_{0}} \frac{d y}{f^{-}(y)}=t & \text { if } t<\tau^{-}\left(x_{0}\right), \\
x(t)=\gamma_{i} & \text { if } t \geq \tau^{-}\left(x_{0}\right) .
\end{array}
$$

We can now combine together the above solutions, and define the semigroup $S$ on the whole real line, as follows.

$$
S_{t}\left(x_{0}\right)=\left\{\begin{array}{ccc}
x_{0} & \text { if } \quad x_{0} \notin\left(\bigcup_{i} I_{i}^{+}\right) \cup\left(\bigcup_{i} I_{i}^{-}\right), \\
S_{t}^{+}\left(x_{0}\right) & \text { if } \quad x_{0} \in\left(\bigcup_{i} I_{i}^{+}\right) \backslash\left(\bigcup_{i} I_{i}^{-}\right), \\
S_{t}^{-}\left(x_{0}\right) & \text { if } \quad x_{0} \in\left(\bigcup_{i} I_{i}^{-}\right) \backslash\left(\bigcup_{i} I_{i}^{+}\right) .
\end{array}\right.
$$

To complete the definition, it remains to define $S_{t}\left(x_{0}\right)$ in the case $x_{0} \in\left(\bigcup_{i} I_{i}^{-}\right) \cap\left(\bigcup_{i} I_{i}^{+}\right)$. Notice that this can happen only if

$$
x_{0}=\alpha_{i}=\delta_{j}
$$

where $I_{i}^{+}=\left[\alpha_{i}, \beta_{i}\left[\right.\right.$ and $\left.\left.I_{j}^{-}=\right] \gamma_{j}, \delta_{j}\right]$ are half-open intervals where the dynamics is increasing and decreasing, respectively. By our definitions, this implies $x_{0} \in \Omega^{*}$. Recalling (Q3), we thus define

$$
S_{t}\left(x_{0}\right)= \begin{cases}S_{t}^{+}\left(x_{0}\right) & \text { if } \quad \Phi\left(x_{0}\right)=1, \\ S_{t}^{-}\left(x_{0}\right) & \text { if } \quad \Phi\left(x_{0}\right)=-1 .\end{cases}
$$

We claim that the above map $S$ defines a semigroup compatible with the ODE (1.1). Toward this goal, we first prove

Lemma 3.1 Let $x:[0, \tau] \mapsto \mathbb{R}$ be a strictly increasing map defined on some interval $[0, \tau]$. Then $x(\cdot)$ is a Carathéodory solution to the Cauchy problem (1.1)-(1.2) if and only if there exists a positive, atomless Borel measure $\mu$, supported on the set $f^{-1}(0)$, such that

$$
\int_{x_{0}}^{x(t)} \frac{1}{f(s)} d s+\mu\left(\left[x_{0}, x(t)\right]\right)=t
$$

for all $t \in[0, \tau]$. 
Proof. 1. Assume that $x(\cdot)$ is a Carathéodory solution to (1.1)-(1.2). Set $x_{0}=x(0)$ and $\widehat{x}=x(\tau)$. Define a positive Borel measure $\mu$ on $\left[x_{0}, \widehat{x}\right]$ by setting

$$
\mu\left(\left[x_{0}, y\right]\right)=\operatorname{meas}(\{t \in[0, t(y)] ; f(x(t))=0\}),
$$

where $t(y)$ is the unique time such that $x(t(y))=y$. Of course, the right hand side of (3.17) refers to Lebesgue measure. Since $f^{-1}(0)$ is closed, for every $\left.x \in\right] x_{0}, \widehat{x}\left[\backslash f^{-1}(0)\right.$ there exists $\delta>0$ such that $] x-\delta, x+\delta[\subset] x_{0}, \widehat{x}\left[\backslash f^{-1}(0)\right.$ and this yields

$$
\mu(] x-\delta, x+\delta[)=0 .
$$

Hence $\mu$ is supported on the set $f^{-1}(0)$. By the continuity and the strict monotonicity property of the function $x(\cdot)$, the map $y \mapsto \mu\left(\left[x_{0}, y\right]\right)$ is continuous. This implies that $\mu$ is an atomless measure.

To prove (3.16) we observe that, for every $t \in[0, \tau]$, the set

$$
\mathcal{T}_{0}(t)=\{s \in[0, t] ; f(x(s))=0\}
$$

is closed. Hence $] 0, t\left[\backslash \mathcal{T}_{0}(t)\right.$ is open and can be written by as a disjoint union of countably many open intervals

$$
] 0, t\left[\backslash \mathcal{T}_{0}(t)=\bigcup_{i}\right] s_{i}, t_{i}[.
$$

By the definitions (3.17)-(3.18) it thus follows

$$
\begin{aligned}
t & =\operatorname{meas}(\{s \in[0, t] ; f(x(s)) \neq 0\})+\operatorname{meas}(\{s \in[0, t] ; f(x(s))=0\}) \\
& =\operatorname{meas}(] 0, t\left[\backslash \mathcal{T}_{0}(t)\right)+\mu\left(\left[x_{0}, x(t)\right]\right) \\
& =\sum_{i} \int_{s_{i}}^{t_{i}} 1 d t+\mu\left(\left[x_{0}, x(t)\right]\right)=\sum_{i} \int_{s_{i}}^{t_{i}} \frac{\dot{x}(t)}{f(x(t))} d t+\mu\left(\left[x_{0}, x(t)\right]\right) \\
& =\sum_{i} \int_{x\left(s_{i}\right)}^{x\left(t_{i}\right)} \frac{1}{f(y)} d y+\mu\left(\left[x_{0}, x(t)\right]\right)=\int_{x_{0}}^{x(t)} \frac{1}{f(y)} d y+\mu\left(\left[x_{0}, x(t)\right]\right),
\end{aligned}
$$

proving (3.16).

2. Conversely, let $\mu$ be a positive, atomless Borel measure on $\left[x_{0}, \widehat{x}\right]=[x(0), x(\tau)]$, and assume that $t \mapsto x(t)$ is a strictly increasing function, implicitly defined by $(3.16)$ for all $t \in[0, \tau]$. For every finite sequence of pairwise disjoint sub-intervals $] s_{i}, t_{i}[\subseteq] 0, \tau[$, we estimate

$$
\sum_{i=1}^{N}\left|t_{i}-s_{i}\right|=\sum_{i=1}^{N}\left(\int_{x\left(s_{i}\right)}^{x\left(t_{i}\right)} \frac{1}{f(y)} d y+\mu\left(\left[x\left(s_{i}\right), x\left(t_{i}\right)\right]\right)\right) \geq \frac{1}{M} \cdot \sum_{i=1}^{N}\left|x\left(t_{i}\right)-x\left(s_{i}\right)\right| .
$$

This implies that $x(\cdot)$ is absolutely continuous on $[0, \tau]$. In particular, $x(\cdot)$ is differentiable almost everywhere in $[0, \tau]$ and

$$
x(t)=x(s)+\int_{s}^{t} \dot{x}(r) d r \quad \text { for all } s<t \in[0, \tau] .
$$


As in (1.4), we denote by $D_{f}$ the set of points where $f$ is discontinuous. For any $\left.s_{0} \in\right] 0, \tau[$ such that $x(\cdot)$ is differentiable at $s_{0}$ and $x\left(s_{0}\right) \notin f^{-1}(0) \cup D_{f}$, one has

$$
\lim _{\varepsilon \rightarrow 0+} \frac{1}{\varepsilon} \cdot \int_{x\left(s_{0}\right)}^{x\left(s_{0}+\varepsilon\right)} \frac{1}{f(y)} d y=\frac{\dot{x}\left(s_{0}\right)}{f\left(x\left(s_{0}\right)\right)} .
$$

Since $\mu$ is supported on the closed set $f^{-1}(0)$, we have $\mu\left(\left[x\left(s_{0}\right), x\left(s_{0}+\delta\right)\right]\right)=0$ for some $\delta>0$. Thus, (3.16) implies

$$
1=\lim _{\varepsilon \rightarrow 0+}\left[\frac{1}{\varepsilon} \cdot \int_{x\left(s_{0}\right)}^{x\left(s_{0}+\varepsilon\right)} \frac{1}{f(y)} d y+\frac{\mu\left(\left[x\left(s_{0}\right), x\left(s_{0}+\varepsilon\right)\right]\right)}{\varepsilon}\right]=\frac{\dot{x}\left(s_{0}\right)}{f\left(x\left(s_{0}\right)\right)} .
$$

Since $D_{f}$ is countable, one has

$$
\dot{x}(s)=f(x(s)) \text { for a.e. } s \in[0, \tau] \backslash \mathcal{T}_{0}(\tau) .
$$

Given any $t \in] 0, \tau[$, the open set $] x(0), x(t)\left[\backslash f^{-1}(0)\right.$ can be written as a disjoint union of countably many open intervals $] \alpha_{k}, \beta_{k}\left[\right.$. Setting $s_{k}=x^{-1}\left(\alpha_{k}\right)$ and $t_{k}=x^{-1}\left(\beta_{k}\right)$, we obtain

$$
\left.\bigcup_{k \geq 1}\right] s_{k}, t_{k}[=] 0, t\left[\backslash \mathcal{T}_{0}(t)\right.
$$

Thus, (3.20) and (3.19) yield

$$
\begin{aligned}
x(t)-x(0) & =\sum_{k \geq 1}\left(x\left(t_{k}\right)-x\left(s_{k}\right)\right)=\sum_{k \geq 1} \int_{s_{k}}^{t_{k}} \dot{x}(s) d s \\
& =\int_{\left.\bigcup_{k \geq 1}\right] s_{k}, t_{k}[} f(x(s)) d s=\int_{] 0, t \backslash \mathcal{T}_{0}(t)} f(x(s)) d s=\int_{0}^{t} f(x(s)) d s
\end{aligned}
$$

showing that $x(\cdot)$ is a Carathéodory solution. This completes the proof.

We are now ready to state the main result of this section.

Theorem 3.1 Let $f: \mathbb{R} \mapsto \mathbb{R}$ satisfy the assumptions (A1)-(A2). The following statements are equivalent.

(i) The map $S: \mathbb{R} \times \mathbb{R}_{+} \rightarrow \mathbb{R}$ is a deterministic semigroup compatible with the ODE (1.1).

(ii) There exist a positive atomless measure $\mu$ supported on the set $f^{-1}(0)$, a countable set of points $\mathcal{S} \subseteq f^{-1}(0)$, and a map $\Phi: \Omega^{*} \mapsto\{-1,1\}$ as in (Q1)-(Q3) such that $S$ coincides with the corresponding semigroup constructed at (3.14)-(3.15).

Proof of the implication (ii) $\Longrightarrow(\mathbf{i})$. Given the measure $\mu$, the set $\mathcal{S}$ and the map $\Phi$, we will show that the map $S: \mathbb{R} \times \mathbb{R}_{+} \mapsto \mathbb{R}$ constructed in (3.14)-(3.15) is indeed a semigroup compatible with the ODE (1.1).

1. Let $x_{0} \in \mathbb{R}$ be given. We first show that the map $t \mapsto x(t)=S_{t}\left(x_{0}\right)$ provides a solution to the Cauchy problem (1.1)-(1.2). Various cases must be considered. 
CASE 1. If $x_{0} \notin\left(\bigcup_{i} I_{i}^{+}\right) \cup\left(\bigcup_{i} I_{i}^{-}\right)$then $S_{t}\left(x_{0}\right)=x_{0}$. In this case we need to show that $f\left(x_{0}\right)=0$. If, on the contrary, $f\left(x_{0}\right) \neq 0$, then by (A2) we would have either

$$
0<\delta \leq f(x) \leq M
$$

for all $\left.\left.x \in \mathcal{N}_{\varepsilon} \doteq\right] x_{0}, x_{0}+\varepsilon\right]$, or else

$$
-M \leq f(x) \leq-\delta<0
$$

for all $x \in \mathcal{N}_{\varepsilon} \doteq\left[x_{0}-\varepsilon, x_{0}[\right.$, for a suitably small $\varepsilon>0$.

We now recall that the measure $\mu$ and the countable set $\mathcal{S}$ in (Q1)-(Q2) satisfy

$$
\operatorname{Supp}(\mu) \cup \mathcal{S} \subseteq f^{-1}(0) .
$$

Since the set $f^{-1}(0)$ is closed and does not contain $x_{0}$, by possibly choosing a smaller $\varepsilon>0$ we can assume that

$$
\mathcal{S} \cap \mathcal{N}_{\varepsilon}=\emptyset, \quad \mu\left(\mathcal{N}_{\varepsilon}\right)=0 .
$$

Together, (3.21)-(3.23) imply that either a strictly increasing trajectory or a strictly decreasing trajectory starts from $x_{0}$. Hence $x_{0} \in\left(\bigcup_{i} I_{i}^{+}\right) \cup\left(\bigcup_{i} I_{i}^{-}\right)$, against the assumption.

CASE 2. Next, assume that $x_{0} \in I_{i}^{+} \backslash\left(\bigcup_{j} I_{j}^{-}\right)$, for some $i \in \mathcal{I}^{+}$and $I_{i}^{+}=\left[\alpha_{i}, \beta_{i}[\right.$. Then the trajectory $t \mapsto x(t)=S_{t}^{+}\left(x_{0}\right)$ is implicitly defined by (3.16). By Lemma 3.1, we already know that $x(\cdot)$ is a Carathéodory solution to the ODE (1.1) for $t \in\left[0, \tau\left(x_{0}\right)\right]$.

It remains to show that, in the case $\tau\left(x_{0}\right)<+\infty$, the function $x(t)=\beta_{i}$ is still a solution of (1.1) for all $t \in\left[\tau\left(x_{0}\right),+\infty\left[\right.\right.$. Of course, this will be the case iff $f\left(\beta_{i}\right)=0$.

Assume that, on the contrary, $f\left(\beta_{i}\right) \neq 0$. Since $f^{-1}(0)$ is closed, there exists a neighborhood $\mathcal{N}$ of $\beta_{i}$ such that (3.23) holds. Moreover, since $f\left(\beta_{i}-\right) \geq 0$, by (A2) it follows $f\left(\beta_{i}+\right)>0$. This implies that, for some $\varepsilon>0$ small enough, the inequalities (3.21) hold for all $x \in \mathcal{N}_{\varepsilon} \doteq$ ]$\left.\beta_{i}, \beta_{i}+\varepsilon\right]$. Moreover, (3.23) holds as well. As a consequence, there exists a strictly increasing trajectory starting at $\beta_{i}$. This yields a contradiction with the maximality of the interval $I_{i}^{+}$.

CASE 3. If $x_{0} \in I_{i}^{-} \backslash\left(\bigcup_{j} I_{j}^{+}\right)$, for some $i \in \mathcal{I}^{-}$, then the same arguments used in Case 2 show that $t \mapsto x(t)=S_{t}^{-}\left(x_{0}\right)$ is a Carathéodory solution.

CASE 4. If $x_{0} \in\left(\bigcup_{i} I_{i}^{-}\right) \cap\left(\bigcup_{i} I_{i}^{+}\right)$, then by (3.15) we are back to Case 2 or Case 3.

2. It remains to prove that the semigroup property $S_{s}\left(S_{t}\left(x_{0}\right)\right)=S_{s+t}\left(x_{0}\right)$ is satisfied.

Referring to the above four cases, in Case 1 this is trivial, because $S_{t}\left(x_{0}\right)=x_{0}$ for all $t \geq 0$.

In Case 2, as long as $t+s \leq \tau^{+}\left(x_{0}\right)$, the semigroup property is a straightforward consequence of the identity

$$
\begin{aligned}
t+s & =\mu\left(\left[x_{0}, x(t+s)\right]\right)+\int_{x_{0}}^{x(t+s)} \frac{d y}{f^{+}(y)} \\
& =\mu\left(\left[x_{0}, x(t)\right]\right)+\mu([x(t), x(t+s)])+\int_{x_{0}}^{x(t)} \frac{d y}{f^{+}(y)}+\int_{x(t)}^{x(t+s)} \frac{d y}{f^{+}(y)} .
\end{aligned}
$$


On the other hand, for $0<t<\tau\left(x_{0}\right) \leq t+s$, the identity

$$
\tau^{+}\left(S_{t}\left(x_{0}\right)\right)=\tau^{+}\left(x_{0}\right)-t
$$

implies

$$
S_{t+s}\left(x_{0}\right)=\beta_{i}=S_{s}\left(S_{t}\left(x_{0}\right)\right) .
$$

Finally, if $t \geq \tau^{+}\left(x_{0}\right)$, then again (3.24) trivially holds.

The remaining Cases 3 and 4 are entirely similar. This concludes the proof of the implication (ii) $\Longrightarrow($ i).

Proof of the implication (i) $\Longrightarrow\left(\right.$ ii). Assuming that $S: \mathbb{R} \times \mathbb{R}_{+} \mapsto \mathbb{R}$ is a deterministic semigroup compatible with the ODE (1.1), in the remaining part of the proof we shall construct a measure $\mu$, a countable set $\mathcal{S}$, and a map $\Phi$ so that $S$ coincides with the corresponding semigroup defined at (3.14)-(3.15).

3. In analogy with Definition 3.1, we introduce:

Definition 3.2 Given the semigroup $S: \mathbb{R} \times \mathbb{R}_{+} \mapsto \mathbb{R}$, we say that an interval $J=[a, b[$ or $J=] a, b\left[\right.$ (open to the right) is a domain of increasing dynamics if, for every $x_{0}, \widehat{x} \in J$ with $x_{0}<\widehat{x}$ there exists $t>0$ such that $\widehat{x}=S_{t} x_{0}$.

Similarly, we say that an interval $J=] a, b]$ or $J=] a, b[$ (open to the left) is a domain of decreasing dynamics if, for every $x_{0}, \widehat{x} \in J$ with $\widehat{x}<x_{0}$ there exists $t>0$ such that $\widehat{x}=S_{t} x_{0}$.

We observe that, if two domains of increasing dynamics $J, J^{\prime}$ have nonempty intersection, then the union $J \cup J^{\prime}$ is also an interval of increasing dynamics. We can thus partition the real line as

$$
\mathbb{R}=\Omega_{S}^{+} \cup \Omega_{S}^{-} \cup \Omega_{S}^{0}
$$

where

- $\Omega_{S}^{+}=\bigcup_{j \in \mathcal{J}^{+}} J_{j}^{+}$is the union of countably many disjoint intervals $J_{j}^{+}=\left[a_{j}, b_{j}\right.$ [ or $\left.J_{j}^{+}=\right] a_{j}, b_{j}$ [ open to the right, where the dynamics is increasing and $b_{j}$ can not be crossed from the left, i.e. $S_{t}\left(b_{j}-\varepsilon\right) \leq b_{j}$ for all $\left.t \geq 0, \varepsilon \in\right] 0, b_{j}-a_{j}[$.

- $\Omega_{S}^{-}=\bigcup_{j \in \mathcal{J}^{-}} J_{j}^{-}$is the union of countably many disjoint intervals $\left.\left.J_{j}^{-}=\right] c_{j}, d_{j}\right]$ or $J_{j}^{-}=$ ] $c_{j}, d_{j}$ [ open to the left, where the dynamics is decreasing and $c_{j}$ can not be crossed from the right, i.e. $S_{t}\left(c_{j}+\varepsilon\right) \geq c_{j}$ for all $\left.t \geq 0, \varepsilon \in\right] 0, d_{j}-c_{j}[$.

- $\Omega_{S}^{0} \subseteq f^{-1}(0)$ is the set of points $x_{0}$ such that $S_{t}\left(x_{0}\right)=x_{0}$ for all $t>0$.

4. We shall construct the measure $\mu$ separately on each interval $J_{j}^{+}, J_{j}^{-}$. As in the proof of Lemma 3.1, we define a positive, Borel measure on $J_{j}^{+}$by setting for every compact subinterval $\left[x_{0}, \widehat{x}\right] \subset J_{j}^{+}$

$$
\mu\left(\left[x_{0}, \widehat{x}\right]\right) \doteq \operatorname{meas}\left(\left\{t \in[0, \tau] ; f\left(S_{t}\left(x_{0}\right)\right)=0\right\}\right)
$$


where $\tau>0$ is such that $\widehat{x}=S_{\tau}\left(x_{0}\right)$. It is clear that $\mu$ is supported on $f^{-1}(0)$. By the semigroup property of $S$, the map $t \mapsto S_{t}\left(x_{0}\right)$ is strictly increasing. Thus, as in the proof of Lemma 3.1, the measure $\mu$ is also atomless. Similarly, one can define the measure $\mu$ on $J_{j}^{-}$.

Next, we define the countable set

$$
\mathcal{S} \doteq\left(\left\{a_{j}, b_{j}: j \in \mathcal{J}^{+}\right\} \cup\left\{c_{j}, d_{j}: j \in \mathcal{J}^{-}\right\}\right) \cap \Omega_{S}^{0}
$$

containing all endpoints of all intervals $J_{j}^{+}, J_{j}^{-}$whose trajectory remains constant.

Finally, the discrete function $\Phi: \Omega^{*} \mapsto\{-1,1\}$ is defined as follows. Let $x_{0} \in \Omega^{*}$. Three cases are considered.

- If $x_{0}=a_{j}$ for some half-open interval $J_{j}^{+}=\left[a_{j}, b_{j}\left[\right.\right.$, then we set $\Phi\left(x_{0}\right)=1$.

- If $x_{0}=d_{j}$ for some half-open interval $\left.\left.J_{j}^{-}=\right] c_{j}, d_{j}\right]$, then we set $\Phi\left(x_{0}\right)=-1$.

- In all other cases, the choice of $\Phi\left(x_{0}\right)$ is immaterial, because it has no effect on the definition of the semigroup. To fix the ideas, we may thus set $\Phi\left(x_{0}\right)=1$.

Recalling Definition 3.1, let $I_{i}^{+}, i \in \mathcal{I}^{+}$, and $I_{i}^{-}, i \in \mathcal{I}^{-}$, be respectively the domains of increase and the domains of decrease corresponding to $\mu, \mathcal{S}$ and $\Phi$. To complete the proof, we first show that

$$
\{] \alpha_{i}, \beta_{i}\left[: i \in \mathcal{I}^{+}\right\}=\{] a_{j}, b_{j}\left[: j \in \mathcal{J}^{+}\right\} \quad \text { and } \quad\{] \gamma_{i}, \delta_{i}\left[: i \in \mathcal{I}^{-}\right\}=\{] c_{j}, d_{j}\left[: j \in \mathcal{J}^{-}\right\}
$$

For any $j \in \mathcal{J}^{+}$, by Lemma 3.1 and (3.26)-(3.27), one has that ] $a_{j}, b_{j}$ [ satisfies (3.6) and this implies that $] a_{j}, b_{j}\left[\subset I_{i}^{+}\right.$for some $i \in \mathcal{I}^{+}$. Moreover, if $a_{j} \in J_{j}^{+}$then $a_{j} \notin \Omega_{S}^{0}$ and

$$
\mu\left(\left[a_{j}, a_{j}+\varepsilon[)+\int_{a_{j}}^{a_{j}+\varepsilon} \frac{1}{f^{+}(y)} d y=t_{\varepsilon}<\infty \quad \text { with } \quad S_{t_{\varepsilon}}\left(a_{j}\right)=a_{j}+\varepsilon\right.\right.
$$

for $0<\varepsilon<b_{j}-a_{j}$. Hence, $a_{j} \in I_{i}^{+}$and this yields $J_{j}^{+} \subseteq I_{i}^{+}$. Furthermore, we prove that $b_{j}=\beta_{i}, a_{j}=\alpha_{i}$ and this implies

$$
] \alpha_{i}, \beta_{i}[=] a_{j}, b_{j}[\text {. }
$$

If $b_{j}<\beta_{i}$ then $b_{j}$ is reachable from the left and the semigroup property of $S$ implies that $b_{j} \in \Omega_{S}^{0}$. In particular, $b_{j} \in \mathcal{S} \cap I_{i}^{+}$and this yields a contradiction. Now assume that $\alpha_{i}<a_{j}$. Two cases are considered:

- If $a_{j} \notin J_{j}^{+}$then $a_{j} \in \Omega_{S}^{0}$ since $\Omega_{S}^{-} \cap I_{i}^{+}=\emptyset$. Thus, $a_{j} \in \mathcal{S} \cap I_{i}^{+}$and this yields a contradiction.

- Otherwise, $a_{j} \in J_{j}^{+}$can not be reached from the left. Thus, either $\mu\left(\left[a_{j}-\varepsilon, a_{j}\right]\right)=+\infty$ for some $\varepsilon \in] 0, a_{j}-\alpha_{i}\left[\right.$ or there exists $b_{j^{\prime}} \in \Omega_{S}^{0} \cap I_{i}^{+}$. Both cases yield a contradiction.

Vice-versa, for any $i \in \mathcal{I}^{+}$, by the definition 3.2 and Lemma 3.1, one has that

$$
\left.\left(\Omega_{S}^{-} \bigcup \operatorname{int}\left(\Omega_{S}^{0}\right)\right) \cap\right] \alpha_{i}, \beta_{i}[=\emptyset .
$$

In particular, $] a_{j}, b_{j}[\cap] \alpha_{i}, \beta_{i}\left[\neq \emptyset\right.$ for some $j \in \mathcal{J}^{+}$. Since $] a_{j}, b_{j}[\subseteq] \alpha_{i^{\prime}}, \beta_{i^{\prime}}$. for some $i^{\prime} \in \mathcal{I}^{+}$ and $\left\{I_{k}\right\}_{k \in \mathcal{I}^{+}}$are disjoint, we have that $] a_{j}, b_{j}[=] \alpha_{i}, \beta_{i}[$, and the first equality of $(3.28)$ holds. Similarly, one can show that the second equality of (3.28). Finally, we need to show that 
(i) If $\alpha_{i} \in I_{i}^{+} \backslash J_{j}^{+}$and $] \alpha_{i}, \beta_{i}[=] a_{j}, b_{j}\left[\right.$ for some $j \in \mathcal{J}^{+}$then

$$
\alpha_{i}=d_{k} \in \Omega^{*} \cap J_{k}^{-} \quad \text { for some } k \in \mathcal{J}^{-} \text {. }
$$

(ii) If $\delta_{i} \in I_{i}^{-} \backslash J_{j}^{-}$and $] \gamma_{i}, \delta_{i}[=] c_{j}, d_{j}\left[\right.$ for some $j \in \mathcal{J}^{-}$then

$$
\delta_{i}=a_{k} \in \Omega^{*} \cap J_{k}^{+} \quad \text { for some } k \in \mathcal{J}^{+} .
$$

Since (i) and (ii) are entirely similar, let us prove (i). Observe that $\alpha_{i}=a_{j}$ can not be in $\Omega_{S}^{0}$, it holds that $\alpha_{i}=d_{k}$ for some $k \in \mathcal{J}^{-}$. On the other hand, one can follow the above argument to show that $J_{k}^{-} \subseteq I_{\ell}^{-}$for some $\ell \in \mathcal{I}^{-}$. Thus, $\alpha_{i}=d_{k} \in I_{i}^{+} \cap I_{\ell}^{-} \subseteq \Omega^{*}$ and this yields (i).

\section{A Markov process whose sample paths solve the ODE}

In this section, we initiate the study of Markov semigroups whose sample paths satisfy the ODE (1.1) with probability one. Our eventual goal is to show that each of these random semigroups can be obtained starting with a deterministic semigroup and performing the two modifications described at (Q4)-(Q5) in the Introduction. In this section, we show that a Markov process can be uniquely determined by the ODE (1.1) and the assignments (Q1)-(Q2) and (Q4)-(Q5), namely:

- A positive, atomless Radon measure supported on $f^{-1}(0)$.

- A countable set $\mathcal{S} \subseteq f^{-1}(0)$ where the dynamics stops.

- A countable set $\mathcal{S}^{*} \subseteq f^{-1}(0)$ and, for each $y_{j} \in \mathcal{S}^{*}$, a number $\lambda_{j}>0$ characterizing the random exponential waiting time, when the trajectories reach the point $y_{j} \in \mathcal{S}^{*}$.

- A map $\Theta: \Omega^{*} \mapsto[0,1]$ determining the probability of moving to the right or to the left, from an initial point $z_{k} \in \Omega^{*}$.

We start by selecting an underlying probability space $\mathcal{W}$ such that, as $\omega \in \mathcal{W}$, the countably many random variables $Y_{j}(\omega) \in \mathbb{R}_{+}$and $Z_{k}(\omega) \in\{-1,1\}$ are independent, with distributions

$$
\text { Prob. }\left\{Y_{j}>s\right\}=e^{-\lambda_{j} s} \quad \text { for all } s>0, \quad \text { Prob. }\left\{Z_{k}=1\right\}=\Theta\left(z_{k}\right) \text {. }
$$

Next, we recall that, given the measure $\mu$ and the countable set $\mathcal{S}$, as in Section 3 one can uniquely determine the sets

$$
\Omega^{+}=\bigcup_{i \in \mathcal{I}^{+}} I_{i}^{+}, \quad \quad \Omega^{-}=\bigcup_{i \in \mathcal{I}^{-}} I_{i}^{-}
$$

consisting of countable unions of disjoint intervals where the dynamics can increase or decrease, respectively. For $x_{0} \in \Omega^{+}$, a trajectory $t \mapsto S_{t}^{+}\left(x_{0}\right)$ was defined at (3.10)-(3.11), while for $x_{0} \in \Omega^{-}$, a trajectory $t \mapsto S_{t}^{-}\left(x_{0}\right)$ was defined at (3.12)-(3.13). 
To construct a Markov process with sample paths $t \mapsto X(t, \omega)$ satisfying (1.1), for every $t>0$ we need to define the transition probabilities

$$
P_{t}\left(x_{0}, A\right)=\operatorname{Prob} .\left\{X(t, \omega) \in A \mid X(0, \omega)=x_{0}\right\},
$$

for any initial point $x_{0} \in \mathbb{R}$ and any Borel set $A \subset \mathbb{R}$. Recalling our construction of a deterministic flow at (3.14)-(3.15), this can be done as follows.

(i) If $x_{0} \notin \Omega^{+} \cup \Omega^{-}$, then $X\left(t, x_{0}, \omega\right)=x_{0}$ for every $t \geq 0$ and $\omega \in \mathcal{W}$. Hence

$$
P_{t}\left(x_{0},\left\{x_{0}\right\}\right)=1 \quad \text { for all } t \geq 0 \text {. }
$$

In other words, all trajectories starting at $x_{0}$ remain constant.

(ii) If $x_{0} \in \Omega^{+} \backslash \Omega^{-}$, a random trajectory starting at $x_{0}$ will have the form

$$
X\left(t, x_{0}, \omega\right)=S_{T^{+}\left(t, x_{0}, \omega\right)}^{+}\left(x_{0}\right),
$$

where the time $T^{+}$along the trajectory is a random variable with distribution

$$
\text { Prob. }\left\{T^{+}\left(t, x_{0}, \omega\right)<s\right\}=\operatorname{Prob} .\left\{s+\sum_{y_{j} \in S^{*} \cap\left[x_{0}, S_{s}^{+}\left(x_{0}\right)\right]} Y_{j}(\omega)>t\right\} .
$$

Note that (4.5) accounts for the (possibly countably many) waiting times when the trajectory crosses one of the points $y_{j} \in \mathcal{S}^{*}$. The transition probabilities are thus given by

$$
\begin{aligned}
& P_{t}\left(x_{0},\right]-\infty, x_{0}[)=0 \\
& P_{t}\left(x_{0},\left[x_{0}, S_{s}^{+}\left(x_{0}\right)\right]\right)=\text { Prob. }\left\{s+\sum_{y_{j} \in S^{*} \cap\left[x_{0}, S_{s}^{+}\left(x_{0}\right)\right]} Y_{j}(\omega) \geq t\right\} .
\end{aligned}
$$

(iii) Similarly, for an initial state $x_{0} \in \Omega^{-} \backslash \Omega^{+}$, a random trajectory starting at $x_{0}$ will have the form

$$
X\left(t, x_{0}, \omega\right)=S_{T^{-}\left(t, x_{0}, \omega\right)}^{-}\left(x_{0}\right),
$$

where the time $T^{-}$along the trajectory is a random variable with distribution

$$
\text { Prob. }\left\{T^{-}\left(t, x_{0}, \omega\right)<s\right\}=\text { Prob. }\left\{s+\sum_{y_{j} \in S^{*} \cap\left[S_{s}^{-}\left(x_{0}\right), x_{0}\right]} Y_{j}(\omega)>t\right\} .
$$

The transition probabilities are thus given by

$$
\begin{aligned}
& P_{t}\left(x_{0},\right] x_{0},+\infty[)=0 \\
& P_{t}\left(x_{0},\left[S_{s}^{-}\left(x_{0}\right), x_{0}\right]\right)=\text { Prob. }\left\{s+\sum_{y_{j} \in S^{*} \cap\left[S_{s}^{-}\left(x_{0}\right), x_{0}\right]} Y_{j}(\omega) \geq t\right\} .
\end{aligned}
$$


(iv) To complete the definition, it remains to define the transition probabilities in the case $x_{0} \in \Omega^{+} \cap \Omega^{-} \subseteq \Omega^{*}$. In this case, by construction we have $x_{0}=z_{k}$ for some $k$. We then define the random variable $X\left(t, x_{0}, \omega\right)$ by setting

$$
X\left(t, x_{0}, \omega\right)= \begin{cases}S_{T^{+}\left(t, x_{0}, \omega\right)}^{+}\left(x_{0}\right) & \text { if } \quad Z_{k}(\omega)=1 \\ S_{T^{-}\left(t, x_{0}, \omega\right)}^{-}\left(x_{0}\right) & \text { if } \quad Z_{k}(\omega)=-1\end{cases}
$$

By (4.1), its distribution satisfies

$$
P_{t}\left(x_{0}, A\right)=\Theta\left(x_{0}\right) \cdot \operatorname{Prob} .\left\{S_{T^{+}\left(t, x_{0}, \omega\right)}^{+} \in A\right\}+\left(1-\Theta\left(x_{0}\right)\right) \cdot \operatorname{Prob} .\left\{S_{T^{-}\left(t, x_{0}, \omega\right)}^{-} \in A\right\}
$$

for every Borel set $A \subseteq \mathbb{R}$.

From the above construction it is clear that, in all cases (i)-(iv), all sample paths $t \mapsto$ $X\left(t, x_{0}, \omega\right)$ are Carathéodory solutions to the Cauchy problem (1.1)-(1.2). Indeed, we are only adding a countable number of waiting times, when the random trajectories reach one of the points $y_{j} \in \mathcal{S}^{*} \subseteq f^{-1}(0)$.

The next result shows that the above transition probabilities define a Markov semigroup.

Proposition 4.1 The transition probability kernels $\left(P_{t}\right)_{t \geq 0}$ in (4.3), (4.6), (4.9), (4.11) define a continuous Markov process. Indeed, they satisfy the Chapman-Kolmogorov equation

$$
\int P_{t}(z, A) P_{s}\left(x_{0}, d z\right)=P_{s+t}\left(x_{0}, A\right)
$$

for all $s, t>0, x_{0} \in \mathbb{R}$ and every Borel set $A \subseteq \mathbb{R}$.

Proof. 1. In Case (i) the conclusion is trivially true. We thus focus on Case (ii). Suppose that $x_{0} \in I_{i}^{+}$for some $i \in \mathcal{I}^{+}$. Observe that $X\left(s, x_{0}, \omega\right)=z \doteq S_{\eta}^{+}\left(x_{0}\right)$ if and only if

$$
\eta+\sum_{y_{j} \in S^{*} \cap\left[x_{0}, S_{\eta}^{+}\left(x_{0}\right)[\right.} Y_{j}(\omega) \leq s \leq \eta+\sum_{y_{j} \in S^{*} \cap\left[x_{0}, S_{\eta}^{+}\left(x_{0}\right)\right]} Y_{j}(\omega)
$$

This leads to two different expressions, depending on whether $z \in S^{*}$ or $z \notin S^{*}$. We claim that, for any Borel set $A \subset \mathbb{R}$,

$$
P_{t+s}\left(x_{0}, A\right)=\sum_{y_{j} \in \mathcal{S}^{*} \cap\left[x_{0}, S_{s}^{+}\left(x_{0}\right)\right]} P_{s}\left(x_{0},\left\{y_{j}\right\}\right) \cdot P_{t}\left(y_{j}, A\right)+\int_{z \in\left[x_{0}, S_{s}^{+}\left(x_{0}\right)\right] \backslash \mathcal{S}^{*}} P_{s}\left(x_{0}, d z\right) P_{t}(z, A) .
$$

It is sufficient to prove (4.14) assuming $x_{0} \in I_{i}^{+}$and $A=\left[x_{0}, b\right] \subseteq I_{i}^{+}$, where $I_{i}^{+}$is one of the intervals in (4.2) where the dynamics is increasing. Consider the times $\eta_{j}, \eta_{z} \geq 0$ such that $y_{j}=S_{\eta_{j}}^{+}\left(x_{0}\right)$ and $z=S_{\eta_{z}}^{+}\left(x_{0}\right)$.

- The time when the random trajectory $t \mapsto X\left(t, x_{0}, \omega\right)$ crosses $z$ is given by

$$
T_{z}^{+}(\omega)=\eta_{z}+\sum_{y_{j} \in S^{*} \cap\left[x_{0}, z\right]} Y_{j}(\omega)
$$


- The times when the random trajectory $t \mapsto X\left(t, x_{0}, \omega\right)$ reaches $y_{j}$, and then leaves $y_{j}$, are given by

$$
T_{j}^{-}(\omega)=\eta_{j}+\sum_{y_{k} \in S^{*} \cap\left[x_{0}, y_{j}[\right.} Y_{k}(\omega), \quad T_{j}^{+}(\omega)=\eta_{j}+\sum_{y_{k} \in S^{*} \cap\left[x_{0}, y_{j}\right]} Y_{k}(\omega) .
$$

In view of the above definition, the left hand side of (4.14) can be written as

$$
\begin{aligned}
& P_{t+s}\left(x_{0},\left[x_{0}, b\right]\right)=\text { Prob. }\left\{T_{b}^{+}(\omega) \geq t+s\right\} \\
& =\text { Prob. }\left\{T_{b}^{+}(\omega) \geq t+s \text { and } X\left(s, x_{0}, \omega\right) \in S^{*}\right\}+\text { Prob. }\left\{T_{b}^{+}(\omega) \geq t+s \text { and } X\left(s, x_{0}, \omega\right) \notin S^{*}\right\} \\
& =\sum_{y_{j} \in S^{*} \cap\left[x_{0}, S_{s}^{+}\left(x_{0}\right)\right]} \text { Prob. }\left\{T_{b}^{+}(\omega) \geq t+s \text { and } X\left(s, x_{0}, \omega\right)=y_{j}\right\} \\
& \quad+\operatorname{Prob} .\left\{T_{b}^{+}(\omega) \geq t+s \text { and } X\left(s, x_{0}, \omega\right) \notin S^{*}\right\}=I+I I .
\end{aligned}
$$

2. For any $y_{j} \in S^{*} \cap\left[x_{0}, S_{s}^{+}\left(x_{0}\right)\right]$, we have

$$
\begin{aligned}
& \text { Prob. }\left\{T_{b}^{+}(\omega) \geq t+s \text { and } X\left(s, x_{0}, \omega\right)=y_{j}\right\} \\
&=\text { Prob. }\left\{T_{b}^{+}(\omega) \geq t+s \text { and } T_{j}^{-}(\omega) \leq s \leq T_{j}^{+}(\omega)\right\} \\
&=\int_{0}^{s} \frac{d}{d r_{1}} \operatorname{Prob} \cdot\left\{T_{j}^{-}(\omega) \in\left[0, r_{1}\right]\right\} \int_{s-r_{1}}^{\infty} \frac{d}{d r_{2}} \operatorname{Prob} \cdot\left\{Y_{j}(\omega) \in\left[0, r_{2}\right]\right\} \\
& \quad \text { Prob. }\left\{t+s-r_{1}-r_{2} \leq T_{b}^{+}(\omega)-T_{j}^{+}(\omega)\right\} d r_{2} d r_{1} \\
&=\int_{0}^{s} \frac{d}{d r_{1}} \operatorname{Prob} \cdot\left\{T_{j}^{-}(\omega) \in\left[0, r_{1}\right]\right\} \\
& \cdot \int_{s-r_{1}}^{\infty} \lambda_{j} e^{-\lambda_{j} r_{2}} \cdot \operatorname{Prob} \cdot\left\{t+s-r_{1}-r_{2} \leq T_{b}^{+}(\omega)-T_{j}^{+}(\omega)\right\} d r_{2} d r_{1} .
\end{aligned}
$$

Performing the change of variable $\tau=r_{2}-\left(s-r_{1}\right)$, one obtains

$$
\begin{aligned}
& \text { Prob. }\left\{T_{b}^{+}(\omega) \geq t+s \text { and } X\left(s, x_{0}, \omega\right)=y_{j}\right\} \\
& =\int_{0}^{s} \frac{d}{d r_{1}} \operatorname{Prob} \cdot\left\{T_{j}^{-}(\omega) \in\left[0, r_{1}\right]\right\} \int_{0}^{\infty} \lambda_{j} e^{-\lambda_{j}\left(\tau+s-r_{1}\right\}} \cdot \operatorname{Prob} \cdot\left\{t-\tau \leq T_{b}^{+}-T_{j}^{+}\right\} d \tau d r_{1} \\
& =\left(\int_{0}^{s} \frac{d}{d r_{1}} \operatorname{Prob} \cdot\left\{T_{j}^{-}(\omega) \in\left[0, r_{1}\right]\right\} \cdot e^{-\lambda_{j}\left(s-r_{1}\right)} d r_{1}\right) \\
& \qquad \cdot\left(\int_{0}^{\infty} \lambda_{j} e^{-\lambda_{j} \tau} \cdot \operatorname{Prob} \cdot\left\{t-\tau \leq T_{b}^{+}(\omega)-T_{j}^{+}(\omega)\right\} d \tau\right) \\
& =\int_{0}^{s} \frac{d}{d r_{1}} \operatorname{Prob} \cdot\left\{T_{j}^{-}(\omega) \in\left[0, r_{1}\right]\right\} \cdot \operatorname{Prob} \cdot\left\{Y_{j}(\omega) \geq s-r_{1}\right\} d r_{1} \\
& \qquad \int_{0}^{\infty} \frac{d}{d \tau} \operatorname{Prob} \cdot\left\{Y_{j}(\omega) \in[0, \tau]\right\} \cdot \operatorname{Prob} \cdot\left\{t-\tau \leq T_{b}^{+}(\omega)-T_{j}^{+}(\omega)\right\} d \tau \\
& =\operatorname{Prob} \cdot\left\{s \in\left[T_{j}^{-}(\omega), T_{j}^{+}(\omega)\right]\right\} \cdot \operatorname{Prob} \cdot\left\{t \leq T_{b}^{+}(\omega)-T_{j}^{-}(\omega)\right\} \\
& =P_{s}\left(x_{0},\left\{y_{j}\right\}\right) \cdot P_{t}\left(y_{j},\left[x_{0}, b\right]\right) .
\end{aligned}
$$


Summing over all $y_{j} \in S^{*}$ we thus obtain

$$
I=\sum_{y_{j} \in S^{*} \cap\left[x_{0}, S_{s}^{+}\left(x_{0}\right)\right]} P_{s}\left(x_{0}, y_{j}\right) \cdot P_{t}\left(y_{j},\left[x_{0}, b\right]\right) .
$$

3. Next, for a fixed integer $N \geq 1$, we consider the following partition of $\left[x_{0}, b\right]$

$$
z_{0} \doteq x_{0}, \quad z_{i} \doteq \sup \left\{z \in\left[x_{0}, b\right]: P_{t}\left(z,\left[x_{0}, b\right]\right) \geq 1-\frac{i}{N}\right\}
$$

and define the open intervals

$$
\left.J_{i} \doteq\right] z_{i-1}, z_{i}[, \quad i \in\{1, \ldots, N\} .
$$

Notice that some of these intervals $J_{i}$ may be empty. For each $i \in\{1, \ldots, N\}$, we estimate

$$
\begin{aligned}
\text { Prob. } & \left\{t+s \leq T_{b}^{+}(\omega) \mid X\left(s, x_{0}, \omega\right) \in J_{i} \backslash S^{*}\right\} \\
& \leq \sup _{z \in J_{i} \backslash S^{*}} \operatorname{Prob} \cdot\left\{t+s \leq T_{b}^{+}(\omega) \mid X\left(s, x_{0}, \omega\right)=z\right\} \\
& =\sup _{z \in J_{i} \backslash S^{*}} \operatorname{Prob} \cdot\left\{t+s \leq T_{b}^{+}(\omega) \mid s=T_{z}^{+}(\omega)\right\}=\sup _{z \in J_{i} \backslash S^{*}} \operatorname{Prob} \cdot\left\{T_{b}^{+}(\omega)-T_{z}^{+}(\omega) \geq t\right\} \\
& =\sup _{z \in J_{i} \backslash S^{*}} P_{t}\left(z,\left[x_{0}, b\right]\right) \leq \inf _{z \in J_{i} \backslash S^{*}} P_{t}\left(z,\left[x_{0}, b\right]\right)+\frac{1}{N} .
\end{aligned}
$$

An entirely similar argument yields

$$
\text { Prob. }\left\{t+s \leq T_{b}^{+}(\omega) \mid X\left(s, x_{0}, \omega\right) \in J_{i} \backslash S^{*}\right\} \geq \sup _{z \in J_{i} \backslash S^{*}} P_{t}\left(z,\left[x_{0}, b\right]\right)-\frac{1}{N} .
$$

Summing over $i=\{1, \ldots, N\}$, one obtains

$$
\begin{aligned}
\text { Prob. }\{t+s \leq & \left.T_{b}^{+}(\omega) \text { and } X\left(s, x_{0}, \omega\right) \notin S^{*}\right\} \\
& =\sum_{i=1}^{N} \text { Prob. }\left\{t+s \leq T_{b}^{+}(\omega) \text { and } X\left(s, x_{0}, \omega\right) \in J_{i} \backslash S^{*}\right\} \\
& =\sum_{i=1}^{N} \operatorname{Prob} .\left\{t+s \leq T_{b}^{+}(\omega) \mid X\left(s, x_{0}, \omega\right) \in J_{i} \backslash S^{*}\right\} \cdot P_{s}\left(x_{0}, J_{i} \backslash S^{*}\right) \\
& \in \sum_{i=1}^{N} \int_{z \in J_{i} \backslash S^{*}} P_{s}\left(x_{0}, d z\right) P_{t}\left(z,\left[x_{0}, b\right]\right)+\left[-\frac{1}{N}, \frac{1}{N}\right] .
\end{aligned}
$$

By letting $N$ to $+\infty$, we thus obtain

$$
I I=\int_{z \in\left[x_{0}, S_{s}^{+}\left(x_{0}\right)\right] \backslash \mathcal{S}^{*}} P_{t}\left(z,\left[x_{0}, b\right]\right) \cdot P_{s}\left(x_{0}, d z\right) .
$$

Together with (4.16) and (4.15), this yields (4.14).

Case (iii) is proved in an analogous way. 
4. In Case (iv), we show that the result follows from the same analysis as in Cases (ii) and (iii). Indeed, it will be sufficient to prove (4.12) for $A=\left[x_{0}, b\right]$, while the case $A=\left[c, x_{0}\right]$ is entirely similar.

If $x_{0} \notin \mathcal{S}^{*}$ then there is no random waiting time at $x_{0}$, and all trajectories immediately start moving (either to the left or to the right of $x_{0}$ ). The same argument used in Case (ii) now yields

$$
\begin{aligned}
\text { Prob. } & \left\{S_{T^{+}\left(t+s, x_{0}, \omega\right)}^{+} \in\left[x_{0}, b\right]\right\} \\
= & \int_{\left[x_{0}, b\right]} \text { Prob. }\left\{S_{T^{+}(t, z, \omega)}^{+} \in\left[x_{0}, b\right]\right\} \cdot \frac{d}{d z} \text { Prob. }\left\{S_{T^{+}\left(s, x_{0}, \omega\right)}^{+} \in\left[x_{0}, z\right]\right\} d z .
\end{aligned}
$$

From (4.11) it thus follows

$$
\begin{aligned}
& \int P_{t}\left(z,\left[x_{0}, b\right]\right) P_{s}\left(x_{0}, d z\right)=\int_{\left[x_{0}, b\right]} P_{t}\left(z,\left[x_{0}, b\right]\right) P_{s}\left(x_{0}, d z\right) \\
& =\Theta\left(x_{0}\right) \cdot \int_{\left[x_{0}, b\right]} \operatorname{Prob} .\left\{S_{T^{+}(t, z, \omega)}^{+} \in\left[x_{0}, b\right]\right\} \cdot \frac{d}{d z} \operatorname{Prob} .\left\{S_{T^{+}\left(s, x_{0}, \omega\right)}^{+} \in\left[x_{0}, z\right]\right\} d z \\
& =\Theta\left(x_{0}\right) \cdot \operatorname{Prob} .\left\{S_{T^{+}\left(t+s, x_{0}, \omega\right)}^{+} \in\left[x_{0}, b\right]\right\}=P_{t+s}\left(x_{0},\left[x_{0}, b\right]\right) .
\end{aligned}
$$

The remaining case is when $x_{0}=y_{j} \in \mathcal{S}^{*}$. We then observe that, for every $\tau \geq 0$, one has

$$
\begin{gathered}
P_{\tau}\left(x_{0},\left\{x_{0}\right\}\right)=\operatorname{Prob} .\left\{Y_{j}(\omega) \geq \tau\right\}=e^{-\lambda_{j} \tau}, \\
P_{\tau}\left(x_{0},\left[x_{0}, b\right]\right)=\Theta\left(x_{0}\right) \cdot \operatorname{Prob} .\left\{S_{T^{+}\left(\tau, x_{0}, \omega\right)}^{+} \in\left[x_{0}, b\right]\right\}+\left(1-\Theta\left(x_{0}\right)\right) \cdot \operatorname{Prob} .\left\{Y_{j}(\omega) \geq \tau\right\} .
\end{gathered}
$$

Using the above identities we obtain

$$
\begin{aligned}
& \int P_{t}\left(z,\left[x_{0}, b\right]\right) P_{s}\left(x_{0}, d z\right)=P_{t}\left(x_{0},\left[x_{0}, b\right]\right) \cdot P_{s}\left(x_{0},\left\{x_{0}\right\}\right)+\int_{] x_{0}, b\right]} P_{t}\left(z,\left[x_{0}, b\right]\right) P_{s}\left(x_{0}, d z\right) \\
&=\quad\left[\left(1-\Theta\left(x_{0}\right)\right) \cdot \operatorname{Prob} .\left\{Y_{j} \geq t\right\}+\Theta\left(x_{0}\right) \cdot \operatorname{Prob} \cdot\left\{S_{T^{+}\left(t, x_{0}, \omega\right)}^{+} \in\left[x_{0}, b\right]\right\}\right] \cdot \operatorname{Prob} .\left\{Y_{j} \geq s\right\} \\
& \quad+\Theta\left(x_{0}\right) \cdot \int_{] x_{0}, b\right]} \operatorname{Prob} \cdot\left\{S_{T^{+}(t, z, \omega)}^{+} \in\left[x_{0}, b\right]\right\} \cdot \frac{d}{d z} \operatorname{Prob} \cdot\left\{S_{T^{+}\left(s, x_{0}, \omega\right)}^{+} \in\left[x_{0}, z\right]\right\} d z \\
&=\left(1-\Theta\left(x_{0}\right)\right) \cdot \operatorname{Prob} \cdot\left\{Y_{j} \geq t+s\right\} \\
& \quad+\Theta\left(x_{0}\right) \cdot \operatorname{Prob} \cdot\left\{S_{T^{+}\left(t, x_{0}, \omega\right)}^{+} \in\left[x_{0}, b\right]\right\} \cdot \operatorname{Prob} \cdot\left\{S_{T^{+}\left(s, x_{0}, \omega\right)}^{+}=x_{0}\right\} \\
& \quad+\Theta\left(x_{0}\right) \cdot \int_{] x_{0}, b\right]} \operatorname{Prob} \cdot\left\{S_{T^{+}(t, z, \omega)}^{+} \in\left[x_{0}, b\right]\right\} \cdot \frac{d}{d z} \operatorname{Prob}\left\{S_{T^{+}\left(s, x_{0}, \omega\right)}^{+} \in\left[x_{0}, z\right]\right\} d z \\
&=\left(1-\Theta\left(x_{0}\right)\right) \cdot \operatorname{Prob} \cdot\left\{S_{T^{-}\left(t+s, x_{0}, \omega\right)}^{-}=x_{0}\right\}+\Theta\left(x_{0}\right) \cdot \operatorname{Prob} \cdot\left\{S_{T^{+}\left(t+s, x_{0}, \omega\right)}^{+} \in\left[x_{0}, b\right]\right\} \\
&= P_{t+s}\left(x_{0},\left[x_{0}, b\right]\right) .
\end{aligned}
$$




\section{Characterization of Markov semigroups compatible with the ODE}

The goal of the present section is to describe the most general Markov semigroup whose sample paths are all solutions to the ODE (1.1). Our main result shows that all of these Markov semigroups are of the form considered in Proposition 4.1. Namely, they are all obtained starting with a deterministic semigroup and performing two modifications: (i) adding a countable number of random waiting times at points $y_{j} \in f^{-1}(0)$, each with a Poisson distribution, and (ii) assigning the probabilities of moving upwards or downwards, at isolated points from where both an increasing and a decreasing solution can initiate.

Theorem 5.1 Let $f$ be a function satisfying(A1)-(A2). The following statements are equivalent.

(I) The random variables $X\left(t, x_{0}, \omega\right)$ yield a Markov process whose sample paths are solutions to the $O D E(1.1)-(1.2)$.

(II) There exist: (i) a positive, atomless Borel measure $\mu$ supported on $f^{-1}(0)$, (ii) a countable set $\mathcal{S} \subseteq f^{-1}(0)$ of stationary points, (iii) a countable set $\mathcal{S}^{*}=\left\{y_{j}: j \geq 1\right\} \subseteq f^{-1}(0)$ and corresponding numbers $\lambda_{j}>0$ determining the Poisson waiting times, and (iv) a map $\Theta: \Omega^{*} \mapsto[0,1]$, such that the transition kernels $P_{t}\left(x_{0}, A\right)=\operatorname{Prob} .\left\{X\left(t, x_{0}, \omega\right) \in A\right\}$ coincide with the corresponding ones constructed at (4.3), (4.6), (4.9), (4.11).

Proof. The implication (II) $\Longrightarrow(\mathrm{I})$ was proved in Proposition 4.1. Here we need to prove $(\mathrm{I}) \Longrightarrow(\mathrm{II})$.

1. We begin by defining two subsets of $f^{-1}(0)$

$$
\begin{aligned}
\Omega_{X}^{0} \doteq\left\{x_{0} ; \text { Prob. }\left\{X\left(1, x_{0}, \omega\right)=x_{0}\right\}=1\right\}, \\
\mathcal{S}^{*} \doteq\left\{x_{0} ; 0<\text { Prob. }\left\{X\left(1, x_{0}, \omega\right)=x_{0}\right\}<1\right\} .
\end{aligned}
$$

Here $\Omega_{X}^{0}$ is the set of points where the motion stops forever, while $\mathcal{S}^{*}$ contains points where the motion stops for a random time, then starts again. By the Markov property and the fact that all solutions of (1.1) are monotone, $x_{0} \in \Omega_{X}^{0}$ implies $X\left(t, x_{0}, \omega\right)=x_{0}$ for all $t \geq 0$ and a.e. $\omega$. We claim that for each $x_{0} \in \mathcal{S}^{*}$, there holds

$$
\text { Prob. }\left\{X\left(t, x_{0}, \omega\right)=x_{0}\right\}=e^{-\lambda t} \quad \text { for all } t \geq 0
$$

with $\lambda \doteq-\log \left(\right.$ Prob. $\left.\left\{X\left(1, x_{0}, \omega\right)=x_{0}\right\}\right)<\infty$. Indeed, for $t \geq 0$, the map

$$
\left.\left.t \mapsto g(t)=\log \left(\operatorname{Prob} .\left\{X\left(t, x_{0}, \omega\right)=x_{0}\right\}\right) \in\right]-\infty, 0\right]
$$

is nonincreasing. By the Markov property, it satisfies

$$
g(0)=0, \quad g(t+s)=g(t)+g(s) \quad \text { for all } t, s>0 .
$$


Therefore, $g(q)=-\lambda q$ for some $\lambda>0$ and all positive rational numbers $q \in \mathbb{Q}_{+}$. Since $g$ is monotone, we conclude that $g$ is continuous on $[0,+\infty[$ and hence

$$
\text { Prob. }\left\{X\left(t, x_{0}, \omega\right)=x_{0}\right\}=e^{g(t)}=e^{-\lambda t} \quad \text { for all } t \in[0,+\infty[\text {. }
$$

2. Next, we need to identify the maximal intervals where the random dynamics is increasing or decreasing.

Definition 5.1 We say that an interval $J=[a, b[$ or $J=] a, b[$ (open to the right) is a domain of increasing random dynamics if for every $x_{0}, \widehat{x} \in J$ with $x_{0}<\widehat{x}$, one has

$$
\lim _{t \rightarrow \infty} \operatorname{Prob} .\left\{X\left(t, x_{0}, \omega\right)>\widehat{x}\right\}=1 .
$$

Similarly, we say that an interval $J=] a, b]$ or $J=] a, b[$ (open to the left) is a domain of decreasing random dynamics if, for every $x_{0}, \widehat{x} \in J$ with $\widehat{x}<x_{0}$, one has

$$
\lim _{t \rightarrow \infty} \operatorname{Prob}\left\{X\left(t, x_{0}, \omega\right)<\widehat{x}\right\}=1 \text {. }
$$

We observe that, if two intervals of increasing random dynamics have nonempty intersection, then their union is still a domain of increasing random dynamics. We can thus define a countable number of maximal open intervals $\left.J_{k}^{+}=\right] a_{k}, b_{k}\left[, k \in \mathcal{J}^{+}\right.$where the random dynamics is increasing, and a countable number of maximal open intervals $\left.J_{k}^{-}=\right] c_{k}, d_{k}\left[, k \in \mathcal{J}^{-}\right.$where the random dynamics is decreasing. Recalling (5.1), we shall consider the countable set

$$
\mathcal{S}=\left(\left\{a_{k}, b_{k}: k \in \mathcal{J}^{+}\right\} \cup\left\{c_{k}, d_{k}: k \in \mathcal{J}^{-}\right\}\right) \cap \Omega_{X}^{0}
$$

Next, we consider the set $\Omega^{*}$ of isolated points from which both a decreasing and an increasing trajectory can initiate. For $z_{k} \in \Omega^{*} \backslash \mathcal{S}$, we define the probability of moving upwards as

$$
\Theta\left(z_{k}\right) \doteq \operatorname{Prob} .\left\{X\left(t, z_{k}, \omega\right)>z_{k} \quad \text { for some } t>0\right\} .
$$

Of course, starting from $z_{k}$, the probability of moving downwards is thus $1-\Theta\left(z_{k}\right)$.

3. Consider a maximal domain of increasing random dynamics, say $J=] a, b[$ or $J=[a, b[$. By definition, for any $a<x<y<b$ we have that

$$
\text { Prob. }\{X(\tau, x, \omega)<y\} \doteq \delta<1
$$

for some $\tau>0$. This implies

$$
\text { Prob. }\{X(k \tau, x, \omega)<y\} \leq \delta^{k} \quad \text { for all } k \geq 1 .
$$

Call $T=T^{x y}>0$ the random time needed for a solution starting at $x$ to reach $y$. By (5.9) it follows

$$
\text { Prob. }\{T>k \tau\} \leq \delta^{k} \text {. }
$$

By (5.10), the probability distribution $T=T^{x y}$ has moments of all orders. In particular, its mean and its variance are finite. 
As a consequence for every $x \leq z<z^{\prime} \leq y$ the random variable $T^{z z^{\prime}}$ has moments of all orders. Its expected value and its variance satisfy

$$
E\left[T^{z z^{\prime}}\right] \leq E\left[T^{x y}\right], \quad \operatorname{Var} .\left(T^{z z^{\prime}}\right) \leq \operatorname{Var} .\left(T^{x y}\right)
$$

Now consider the points $y_{j} \in[x, y]$ where a random waiting time $Y_{j}$ occurs. The Markov property implies that $Y_{j}$ has a Poisson distribution as in (4.1), with expected value $E\left[Y_{j}\right]=\frac{1}{\lambda_{j}}$. From (5.11), it follows that for every finite subset $S_{1}^{*}$ of $S^{*} \cap[x, y]$

$$
\sum_{y_{j} \in \mathcal{S}_{1}^{*}} \frac{1}{\lambda_{j}}=\sum_{y_{j} \in \mathcal{S}_{1}^{*}} E\left[Y_{j}\right] \leq E\left[T^{x y}\right]<+\infty .
$$

In particular,

$$
\#\left\{y_{j} \in \mathcal{S}^{*} \cap[x, y]: \lambda_{j} \leq n\right\} \leq n \cdot E\left[T^{x y}\right] \quad \text { for all } n \geq 1
$$

and this shows that the set $\mathcal{S}^{*}$ of points where a random waiting time occurs is at most countable. Thus, we can write $\mathcal{S}^{*} \cap J=\left\{y_{1}, y_{2}, \ldots\right\}$ and

$$
\sum_{z \leq y_{j}<z^{\prime}} \frac{1}{\lambda_{j}}=\sum_{z \leq y_{j}<z^{\prime}} E\left[Y_{j}\right] \leq E\left[T^{z z^{\prime}}\right] .
$$

4. To help the reader, we provide here an outline the remaining steps of the proof. Let $J=] a, b[$ or $J=\left[a, b\left[\right.\right.$ be a maximal domain of increasing random dynamics and $\left\{y_{1}, y_{2}, \ldots\right\}=J \cap \mathcal{S}^{*}$ be the set of points where the trajectories stop for a random time $Y_{j}$, then start again. We can then define a new Markov semigroup by removing these waiting times. More precisely, for each $n \geq 1$, we define a Markov semigroup $S^{(n)}$ whose trajectories $t \mapsto X^{(n)}\left(t, x_{0}, \omega\right)$ satisfy

$$
X^{(n)}\left(\tau_{n}\left(t, x_{0}, \omega\right), x_{0}, \omega\right)=X\left(t, x_{0}, \omega\right),
$$

where

$$
\tau_{n}\left(t, x_{0}, \omega\right) \doteq t-\operatorname{meas}\left\{s \in[0, t] ; X\left(s, x_{0}, \omega\right) \in\left\{y_{1}, \ldots, y_{n}\right\}\right\} .
$$

In turn, given $S^{(n)}$, we can recover the original semigroup $S$ by inserting back the waiting times at the points $y_{1}, \ldots, y_{n}$.

Now consider the limit semigroup $\widetilde{S}=\lim _{n \rightarrow \infty} S^{(n)}$. Since we removed all the random waiting times, restricted to the interval $J$ the trajectories of $\widetilde{S}$ are strictly increasing with probability one. This will imply that $\widetilde{S}$ is a deterministic semigroup, hence it admits the representation proved in Theorem 3.1. Namely, there exists a positive atomless measure $\mu$ on $J \cap f^{-1}(0)$ such that trajectories of $\widetilde{S}$ are determined by the formula (3.11). In turn, the original Markov semigroup $S$ can be recovered from $\widetilde{S}$ by adding back the random waiting times $Y_{j}$ at the points $y_{j}$. This will provide the desired characterization of $S$.

5. We now work out details. Consider the first point $y_{1}$. We claim that, starting with the Markov semigroup $S$ and removing the random waiting time $Y_{1}(\omega)$ at $y_{1}$, we obtain another Markov semigroup $S^{(1)}$. 
Indeed, given an initial point $x_{0} \in J$, let $t \mapsto X\left(t, x_{0}, \omega\right)$ be a sample path of the original process, and define the new path $X^{(1)}\left(\cdot, x_{0}, \omega\right)$ by setting

$$
X^{(1)}\left(t, x_{0}, \omega\right)= \begin{cases}X\left(t, x_{0}, \omega\right) & \text { if } \left.\quad X\left(t, x_{0}, \omega\right) \in\right] a, y_{1}\left[\text { or } x_{0}>y_{1},\right. \\ X\left(t+Y_{1}(\omega), x_{0}, \omega\right) & \text { if } \quad X\left(t, x_{0}, \omega\right) \in\left[y_{1}, b\left[\text { and } x_{0} \leq y_{1}\right.\right.\end{cases}
$$

We claim that the transition probability kernels

$$
P_{\tau}^{(1)}\left(x_{0}, A\right) \doteq \operatorname{Prob} .\left\{X^{(1)}\left(\tau, x_{0}, \omega\right) \in A\right\}
$$

satisfy the Chapman-Kolmogorov equation. Indeed, for any Borel set $B \subset] a, b[$, the following holds.

If $x_{0}>y_{1}$, then

$$
P_{t}^{(1)}\left(x_{0}, B\right)=P_{t}\left(x_{0}, B\right) .
$$

Otherwise, if $x_{0} \leq y_{1}$, setting $\left.B_{1}=\right] a, y_{1}\left[\cap B\right.$ and $B_{2}=\left[y_{1}, b[\cap B\right.$, one obtains

$$
\begin{aligned}
P_{t}^{(1)}\left(x_{0}, B\right) & =\operatorname{Prob} .\left\{X\left(t, x_{0}, \omega\right) \in B_{1}\right\}+\operatorname{Prob} .\left\{X\left(t+Y_{1}(\omega), x_{0}, \omega\right) \in B_{2}\right\} \\
& =P_{t}\left(x_{0}, B_{1}\right)+\int_{0}^{\infty} \text { Prob. }\left\{X\left(t+\tau, x_{0}, \omega\right) \in B_{2}\right\} \lambda_{1} e^{-\lambda_{1} \tau} d \tau \\
& =P_{t}\left(x_{0}, B_{1}\right)+\int_{0}^{\infty} P_{t+\tau}\left(x_{0}, B_{2}\right) \cdot \lambda_{1} e^{-\lambda_{1} \tau} d \tau .
\end{aligned}
$$

Therefore, for any $s, t>0$ and any Borel set $A \subseteq] a, b\left[\right.$, if $x_{0}>y_{1}$ then we trivially have

$$
\int P_{t}^{(1)}(z, A) P_{s}^{(1)}\left(x_{0}, d z\right)=\int P_{t}(z, A) P_{s}\left(x_{0}, d z\right)=P_{t+s}\left(x_{0}, A\right)=P_{t+s}^{(1)}\left(x_{0}, A\right) .
$$

Otherwise, if $x_{0} \leq y_{1}$, then we consider the sets $\left.A_{1}=A \cap\right] a, y_{1}\left[, A_{2}=A \cap\left[y_{1}, b[\right.\right.$, and compute

$$
\begin{aligned}
\int P_{t}^{(1)}(z, A) P_{s}^{(1)}\left(x_{0}, d z\right) & \\
& =\int P_{t}\left(z, A_{1}\right) P_{s}\left(x_{0}, d z\right)+\int P_{t}\left(z, A_{2}\right) \cdot\left[\int_{0}^{\infty} P_{s+\tau}\left(x_{0}, d z\right) \cdot \lambda_{1} e^{-\lambda_{1} \tau} d \tau\right] \\
& =P_{t+s}\left(x_{0}, A_{1}\right)+\int_{0}^{\infty}\left[\int P_{t}\left(z, A_{2}\right) \cdot P_{s+\tau}\left(x_{0}, d z\right)\right] \cdot \lambda_{1} e^{-\lambda_{1} \tau} d \tau \\
& =P_{t+s}\left(x_{0}, A_{1}\right)+\int_{0}^{\infty} P_{t+s+\tau}\left(x_{0}, A_{2}\right) \cdot \lambda_{1} e^{-\lambda_{1} \tau} d \tau=P_{t+s}^{(1)}\left(x_{0}, A\right) .
\end{aligned}
$$

Hence, the process $S^{(1)}$ with sample paths $t \mapsto X^{(1)}\left(t, x_{0}, \omega\right)$ is a Markov semigroup.

6. By induction, for each $n \geq 1$, we consider the process $S^{(n)}$ obtained from $S^{(n-1)}$ by removing the Poisson waiting time at the point $y_{n} \in J \cap \mathcal{S}^{*}$. By the previous argument, every $S^{(n)}$ is a Markov semigroup. Conversely, one can recover the original Markov semigroup $S$ starting with $S^{(n)}$ and adding the Poisson waiting times $Y_{1}, \ldots, Y_{n}$ at the points $y_{1}, \ldots, y_{n}$. If the set $J \cap \mathcal{S}^{*}$ contains $N$ points, the induction argument is concluded $N$ steps. Otherwise, since the sequence of trajectories $t \mapsto X^{(n)}\left(t, x_{0}, \omega\right)$ is monotone increasing and bounded for $t$ in bounded sets, for every $\omega$ there exists the limit $X^{(n)}\left(t, x_{0}, \omega\right) \rightarrow \widetilde{X}\left(t, x_{0}, \omega\right)$, uniformly for 
$t$ in bounded sets. By part (iii) of Theorem 2.1, each limit trajectory $t \mapsto \widetilde{X}\left(t, x_{0}, \omega\right)$ is still a solution of the ODE (1.1).

In the next step we will prove that this limit process is deterministic. Namely

$$
\operatorname{Var} .\left(\widetilde{X}\left(t, x_{0}, \omega\right)\right)=0
$$

for every $x_{0} \in J$ and $t>0$.

7. Given $x_{0}<\widehat{x} \in J$, let us define

$$
\left\{\begin{array}{l}
T_{n}^{x_{0} \widehat{x}}(\omega) \doteq \inf \left\{t>0 ; \quad X^{(n)}\left(t, x_{0}, \omega\right) \geq \widehat{x}\right\} \\
\widetilde{T}^{x_{0} \widehat{x}}(\omega) \doteq \inf \left\{t>0 ; \quad \widetilde{X}\left(t, x_{0}, \omega\right) \geq \widehat{x}\right\}
\end{array}\right.
$$

the random times needed to reach $\widehat{x}$, for a trajectory starting at $x_{0}$, having removed respectively the first $n$ waiting times at $y_{1}, \ldots, y_{n}$ or all waiting times $y_{j} \in J \cap S^{*}$.

Let $\varepsilon_{0}>0$ be such that $E\left[\widetilde{T}^{x_{0} \widehat{x}}\right]>\varepsilon_{0}$. By $(5.12)$ we can choose $n$ large enough so that

$$
\sum_{j>n, x_{0} \leq y_{j}<\widehat{x}} \frac{1}{\lambda_{j}}<\varepsilon_{0} .
$$

We now consider the expected values of these random times. By construction the map $y \mapsto$ $E\left[T_{n}^{x_{0} y}\right]$ is nondecreasing, and has upward jumps in the amount $\frac{1}{\lambda_{j}}$ at each point $y_{j}$, with $j>n$. By (5.14), we can introduce a partition

$$
x=x_{0}<x_{1}<\cdots<x_{\nu}=\widehat{x}
$$

such that

$$
\varepsilon_{0} \leq E\left[T_{n}^{x x_{i}}\right]-E\left[T_{n}^{x x_{i-i}}\right]=E\left[T_{n}^{x_{i-1} x_{i}}\right] \leq 2 \varepsilon_{0} \quad \text { for all } i=1,2, \ldots, \nu .
$$

Notice that, setting

$$
K \doteq E\left[T_{n}^{x_{0} \widehat{x}}\right]
$$

the inequalities (5.15) require

$$
\frac{K}{2 \epsilon_{0}} \leq \nu \leq \frac{K}{\epsilon_{0}}
$$

From (5.15) it follows

$$
2 \varepsilon_{0} \geq E\left[T_{n}^{x_{i-1} x_{i}}\right] \geq \frac{4 K}{\nu} \cdot \operatorname{Prob} \cdot\left\{T_{n}^{x_{i-1} x_{i}} \geq \frac{4 K}{\nu}\right\},
$$

and (5.16) implies

$$
\text { Prob. }\left\{X^{(n)}\left(\frac{4 K}{\nu}, x_{i-1}, \omega\right)<x_{i}\right\}=\text { Prob. }\left\{T_{n}^{x_{i-1} x_{i}} \geq \frac{4 K}{\nu}\right\} \leq \frac{1}{2} \cdot \frac{\nu \varepsilon_{0}}{K} \leq \frac{1}{2} .
$$

In turn, for every $j \geq 1$, it holds

$$
\text { Prob. }\left\{T_{n}^{x_{i-1} x_{i}} \geq \frac{4 K j}{\nu}\right\}=\text { Prob. }\left\{X^{(n)}\left(\frac{4 j K}{\nu}, x_{i-1}, \omega\right)<x_{i}\right\} \leq 2^{-j} .
$$


Let us now estimate the variance of the random time $\widetilde{T}^{x_{i-1} x_{i}}$ needed to move from $x_{i-1}$ to $x_{i}$. Call

$$
\Phi(s) \doteq \operatorname{Prob} .\left\{\widetilde{T}^{x_{i-1} x_{i}}>s\right\} .
$$

Observing that $\Phi$ is a non-increasing function which satisfies

$$
\Phi(4 j K / \nu) \leq \text { Prob. }\left\{T_{n}^{x_{i-1} x_{i}} \geq \frac{4 K j}{\nu}\right\} \leq 2^{-j} \quad \text { for all } j \geq 1,
$$

we estimate the variance ${ }^{1}$

$$
\begin{aligned}
\operatorname{Var} .\left(\widetilde{T}^{x_{i-1} x_{i}}\right) & \leq-\int_{0}^{+\infty} s^{2} d \Phi(s)=\int_{0}^{+\infty} 2 s \Phi(s) d s \\
& =\sum_{j \geq 1} \int_{4(j-1) K / \nu}^{4 j K / \nu} 2 s \Phi(s) d s \leq \frac{4 K}{\nu} \sum_{j \geq 1} \frac{4 j K}{\nu} 2^{1-j}=\frac{64 K^{2}}{\nu^{2}} .
\end{aligned}
$$

We observe that, for $i=1, \ldots, \nu$, the random variables $\widetilde{T}^{x_{i-1} x_{i}}$ are mutually independent. Hence, by (5.16),

$$
\operatorname{Var} .\left(\widetilde{T}^{x_{0} \widehat{x}}\right)=\sum_{i=1}^{\nu} \operatorname{Var} .\left(\widetilde{T}^{x_{i-1} x_{i}}\right) \leq \sum_{i=1}^{\nu} \frac{64 K^{2}}{\nu^{2}} \leq \frac{64 K^{2}}{\nu} \leq 128 K \varepsilon_{0} .
$$

Since $\varepsilon_{0}>0$ can be chosen arbitrarily small, we conclude that the variance of $\widetilde{T}^{x_{0} \widehat{x}}$ is zero. Hence, the limit process is deterministic.

8. We now define

$$
\widetilde{S}_{t} x_{0} \doteq \lim _{n \rightarrow \infty} E\left[X^{(n)}\left(t, x_{0}, \omega\right)\right] .
$$

The existence of the limit follows trivially by monotonicity. Since the variance of the random variables $X^{(n)}$ goes to zero, again by (iii) in Theorem 2.1 it follows that each trajectory $t \mapsto \widetilde{S}_{t} x_{0}$ is a Carathéodory solution to (1.1).

We claim that $\widetilde{S}$ is a deterministic semigroup. Toward this goal, we first observe that the maps

$$
t \mapsto \widetilde{S}_{t} x_{0}, \quad x_{0} \mapsto \widetilde{S}_{t} x_{0}
$$

are both Lipschitz, and strictly increasing (as long as $\widetilde{S}_{t} x_{0}<b$ ). To prove the semigroup identity

$$
\widetilde{S}_{s}\left(\widetilde{S}_{t} x_{0}\right)=\widetilde{S}_{t+s} x_{0},
$$

set $x_{1}=\widetilde{S}_{t} x_{0}$ and let $\varepsilon>0$ be given. Choose $\delta>0$ such that

$$
\widetilde{S}_{s}\left(x_{1}-\delta\right)>\widetilde{S}_{s} x_{1}-\varepsilon, \quad \widetilde{S}_{s}\left(x_{1}+\delta\right)<\widetilde{S}_{s} x_{1}+\varepsilon
$$

We now have

$$
\lim _{n \rightarrow \infty} \operatorname{Prob} .\left\{X^{(n)}\left(t, x_{0}, \omega\right) \in\left[x_{1}-\delta, x_{1}+\delta\right]\right\}=1
$$

\footnotetext{
${ }^{1}$ Indeed, differentiating the identity $\frac{1}{1-x}=\sum_{j=0}^{\infty} x^{j}$ one obtains $\frac{1}{(1-x)^{2}}=\sum_{j=1}^{\infty} j x^{j-1}$. Setting $x=1 / 2$ we get $\sum_{j=1}^{\infty} j 2^{1-j}=4$
} 
By (5.21) and a comparison argument, this implies

$$
\lim _{n \rightarrow \infty} \text { Prob. }\left\{X^{(n)}\left(t+s, x_{0}, \omega\right) \in\left[\widetilde{S}_{s} x_{1}-\varepsilon, \widetilde{S}_{s} x_{1}+\varepsilon\right]\right\}=1 .
$$

Since $\varepsilon>0$ was arbitrary, this proves the semigroup property (5.20).

As a consequence, by Theorem 3.1, there exists a positive atomless measure $\mu$ supported on $J \cap f^{-1}(0)$ such that the trajectories of the semigroup are characterized by the formula (3.11).

Of course, the above construction of a measure $\mu$ can be repeated on every maximal interval $J$ of increasing or decreasing dynamics.

9. As in Proposition 4.1, given the sets $\mathcal{S}, \mathcal{S}^{*} \subseteq f^{-1}(0)$, the positive, atomless measure $\mu$ on $f^{-1}(0)$, and the maps $\Lambda: \mathcal{S}^{*} \mapsto \mathbb{R}_{+}, \Theta: \Omega^{*} \mapsto[0,1]$, we construct a unique Markov semigroup $\widehat{S}$, with transition probabilities given at (4.3), (4.6), (4.9), (4.11). Recalling Definition 3.1, let $I_{i}^{+}, i \in \mathcal{I}^{+}$, and $I_{i}^{-}, i \in \mathcal{I}^{-}$, be respectively the domains of increase and the domains of decrease corresponding to $\mu$ and $\mathcal{S}$. For any $k \in \mathcal{J}^{+}$and $x_{0} \in J_{k}^{+}$with $\left.J_{k}^{+}=\right] a_{k}, b_{k}$ [ or $J_{k}^{+}=\left[a_{k}, b_{k}\left[\right.\right.$, the map $\left[0, T_{b_{k}}\left[\ni t \mapsto \widetilde{S}_{t} x_{0}\right.\right.$ is strictly increasing and $\lim _{t \rightarrow T_{b_{k}}} \widetilde{S}_{t} x_{0}=b_{k}$ for some $T_{b_{k}}>0$. Thus, $J_{k}^{+}$is an strictly increasing domain associated to $\widetilde{S}$ and this implies that

$$
J_{k}^{+} \subseteq I_{i}^{+} \quad \text { for some } i \in \mathcal{I}^{+} .
$$

Similarly, for every $h \in \mathcal{J}^{-}$it holds that $J_{h}^{-} \subseteq I_{j}^{-}$for some $i \in \mathcal{I}^{-}$. Thus,

$$
\bigcup_{k \in \mathcal{J}^{+}} J_{k}^{+} \subseteq \bigcup_{i \in \mathcal{I}^{+}} I_{i}^{+} \quad \text { and } \quad \bigcup_{h \in \mathcal{J}^{-}} J_{h}^{-} \subseteq \bigcup_{i \in \mathcal{I}^{-}} I_{i}^{-}
$$

In the remainder of the proof, we will show that the given Markov semigroup $S$ coincides with the semigroup $\widehat{S}$ constructed in Proposition 4.1. In other words, for every $x_{0} \in \mathbb{R}, t>0$ and any Borel set $A \subset \mathbb{R}$, the transition probabilities coincide:

$$
P_{t}\left(x_{0}, A\right)=\widehat{P}_{t}\left(x_{0}, A\right) \doteq \operatorname{Prob} .\left\{\widehat{S}_{t} x_{0} \in A\right\} .
$$

Four cases must be considered:

(i) $x_{0} \notin\left(\bigcup_{i \in \mathcal{I}^{+}} I_{i}^{+}\right) \cup\left(\bigcup_{i \in \mathcal{I}^{-}} I_{i}^{-}\right)$;

(ii) $x_{0} \in\left(\bigcup_{i \in \mathcal{I}^{+}} I_{i}^{+}\right) \backslash\left(\bigcup_{i \in \mathcal{I}^{-}} I_{i}^{-}\right)$;

(iii) $x_{0} \in\left(\bigcup_{i \in \mathcal{I}^{-}} I_{i}^{-}\right) \backslash\left(\bigcup_{i \in \mathcal{I}^{+}} I_{i}^{+}\right)$;

(iv) $x_{0} \in\left(\bigcup_{i \in I^{-}} I_{i}^{-}\right) \cap\left(\bigcup_{i \in \mathcal{I}^{+}} I_{i}^{+}\right) \subseteq \Omega^{*}$.

Case (i) is trivial. We thus focus on Case (ii). Assume that $x_{0} \in I_{i}^{+} \backslash\left(\bigcup_{j} I_{j}^{-}\right)$for some $i \in \mathcal{I}^{+}$. In this case, we only need to prove (5.22) for $A=\left[x_{0}, \widetilde{S}_{s}\left(x_{0}\right)\right]$ with $s>0$. From the previous construction in Proposition 4.1, it holds that

$$
\widehat{P}_{t}\left(x_{0},\left[x_{0}, \widetilde{S}_{s}\left(x_{0}\right)\right]\right)=\text { Prob. }\left\{s+\sum_{y_{j} \in S^{*} \cap\left[x_{0}, \widetilde{S}_{s}\left(x_{0}\right)\right]} Y_{j}(\omega) \geq t\right\} .
$$

Two cases are considered: 
- If $x_{0} \in J_{k}^{+}$for some $k \in \mathcal{J}^{+}$then one has

$$
\begin{aligned}
& P_{t}\left(x_{0},\left[x_{0}, \widetilde{S}_{s}\left(x_{0}\right)\right]\right)=\operatorname{Prob} .\left\{X\left(t, x_{0}, \omega\right) \in\left[x_{0}, \widetilde{S}_{s}\left(x_{0}\right)\right]\right\} \\
& \quad=\operatorname{Prob} .\left(\widetilde{X}\left(\tau\left(t, x_{0}, \omega\right), x_{0}, \omega\right) \in\left[x_{0}, \widetilde{X}\left(s, x_{0}, \omega\right)\right]\right) \\
& \quad=\operatorname{Prob} .\left(\tau\left(t, x_{0}, \omega\right) \leq s\right)=\operatorname{Prob} .\left\{s+\sum_{y_{j} \in S^{*} \cap\left[x_{0}, \widetilde{S}_{s}\left(x_{0}\right)\right]} Y_{j}(\omega) \geq t\right\},
\end{aligned}
$$

where

$$
\tau\left(t, x_{0}, \omega\right) \doteq t-\operatorname{meas}\left\{r \in[0, t] ; X\left(r, x_{0}, \omega\right) \in \mathcal{S}^{*}\right\} .
$$

This yields (5.22) for $A=\left[x_{0}, \widetilde{S}_{s}\left(x_{0}\right)\right]$.

- Otherwise, if $x_{0} \notin \bigcup_{k \in \mathcal{J}^{+}} J_{k}^{+}$then

$$
\lim _{t \rightarrow \infty} \operatorname{Prob} .\left\{X\left(t, x_{0}, \omega\right) \leq x\right\}=1 \quad \text { for all } x>x_{0} .
$$

and this implies that

$$
\text { Prob. }\left\{S_{t} x_{0}=x_{0}\right\}=1 \quad \text { and } \quad x_{0} \in \Omega_{X}^{0} .
$$

Indeed, assume by a contradiction that there exists $\widehat{x}>x_{0}$ such that Prob. $\left\{X\left(\widehat{t}, x_{0}, \omega\right) \leq\right.$ $\widehat{x}\}=\delta<1$ for some $\widehat{t}>0$. By the Markov property of $X$, we have

$$
\text { Prob. }\left\{X\left(k \widehat{t}, x_{0}, \omega\right) \leq \widehat{x}\right\} \leq \delta^{k} \quad \text { for all } k \in \mathbb{Z}^{+} .
$$

In particular, the monotone increasing property of the maps $t \mapsto X\left(t, x_{0}, \omega\right)$ implies that $\lim _{t \rightarrow \infty}$ Prob. $\left\{X\left(\widehat{t}, x_{0}, \omega\right) \leq \widehat{x}\right\}=0$. Hence, $x_{0}$ is in a domain of increasing random dynamics and this yields a contradiction.

On the other hand, since $x_{0} \notin\left(\bigcup_{k \in \mathcal{J}^{+}} J_{k}^{+}\right) \bigcup\left(\bigcup_{j} I_{j}^{-}\right)$and $\bigcup_{h \in \mathcal{J}^{-}} J_{h}^{-} \subseteq \bigcup_{i \in \mathcal{I}^{-}} I_{i}^{-}$, one has that $x_{0} \in\left(\left\{a_{k}, b_{k}: k \in \mathcal{J}^{+}\right\} \cup\left\{c_{k}, d_{k}: k \in \mathcal{J}^{-}\right\}\right)$. Thus, (5.25) and (4.3) implies that $x_{0} \in \mathcal{S}$ and

$$
\widehat{P}_{t}\left(x_{0},\left\{x_{0}\right\}\right)=1=P_{t}\left(x_{0},\left\{x_{0}\right\}\right) \text {. }
$$

Similarly, one can prove (5.22) for $x_{0}$ in an interval of decreasing dynamics.

Finally, consider $x_{0} \in\left(I_{i}^{+} \cap I_{i^{\prime}}^{-}\right) \backslash \mathcal{S}$, for some $i \in \mathcal{I}^{+}$and $i^{\prime} \in \mathcal{I}^{-}$. It is sufficient to verify (5.22) for $A=\left[x_{0}, b\right]$, with $b \in I_{i}^{+}$. The case $A=\left[c, x_{0}\right], c \in I_{i^{\prime}}^{-}$is entirely similar. If $x_{0} \notin \mathcal{S}^{*}$ then

$$
\text { Prob. }\left\{X\left(1, x_{0}, \omega\right)=x_{0}\right\}=0 \quad \text { and } \quad \text { Prob. }\left\{X\left(1, x_{0}, \omega\right)>x_{0}\right\}=\Theta\left(x_{0}\right)
$$

and this implies

$$
\begin{aligned}
\text { Prob. } & \left\{X\left(t, x_{0}, \omega\right) \in\left[x_{0}, b\right]\right\}=\text { Prob. }\left\{X\left(t, x_{0}, \omega\right) \in\left[x_{0}, b\right] \text { and } X\left(1, x_{0}, \omega\right)>x_{0}\right\} \\
= & \text { Prob. }\left\{X\left(1, x_{0}, \omega\right)>x_{0}\right\} \cdot \text { Prob. }\left\{X\left(t, x_{0}, \omega\right) \in\left[x_{0}, b\right] \mid X\left(1, x_{0}, \omega\right)>x_{0}\right\} \\
= & \Theta\left(x_{0}\right) \cdot \operatorname{Prob} .\left\{\widetilde{S}_{T^{+}\left(t, x_{0}, \omega\right)}^{+} \in\left[x_{0}, b\right]\right\}=\widehat{P}_{t}\left(x_{0},\left[x_{0}, b\right]\right) .
\end{aligned}
$$


Otherwise, if $x_{0}=y_{j} \in \mathcal{S}^{*}$ then

$$
\begin{aligned}
\text { Prob. }\left\{X\left(t, x_{0}, \omega\right) \in\left[x_{0}, b\right]\right\} & \\
=\text { Prob. }\left\{X\left(t, x_{0}, \omega\right) \in\left[x_{0}, b\right] \text { and } X\left(s, x_{0}, \omega\right)>x_{0} \text { for some } s>0\right\} & \quad \text { Prob. }\left\{X\left(t, x_{0}, \omega\right) \in\left[x_{0}, b\right] \text { and } X\left(s, x_{0}, \omega\right)<x_{0} \text { for some } s>0\right\} \\
= & \left.\Theta\left(x_{0}\right) \cdot \operatorname{Prob} .\left\{\widetilde{S}_{T^{+}\left(t, x_{0}, \omega\right)}^{+} \in\left[x_{0}, b\right]\right\}\right\} \\
& +\left(1-\Theta\left(x_{0}\right)\right) \cdot \operatorname{Prob} \cdot\left\{X\left(t, x_{0}, \omega\right)=x_{0} \mid X\left(s, x_{0}, \omega\right)<x_{0} \text { for some } s>0\right\} \\
= & \Theta\left(x_{0}\right) \cdot \operatorname{Prob} \cdot\left\{S_{T^{+}\left(t, x_{0}, \omega\right)}^{+} \in\left[x_{0}, b\right]\right\}+\left(1-\Theta\left(x_{0}\right)\right) \cdot \operatorname{Prob} .\left\{Y_{j}(\omega) \geq t\right\} \\
= & \widehat{P}_{t}\left(x_{0},\left[x_{0}, b\right]\right) .
\end{aligned}
$$

This establishes the identity (5.22) for all initial points $x_{0} \in \mathbb{R}$, completing the proof of the theorem.

Acknowledgments. This research by K. T. Nguyen was partially supported by a grant from the Simons Foundation/SFARI (521811, NTK).

\section{References}

[1] G. Alberti, S. Bianchini, and G. Crippa, A uniqueness result for the continuity equation in two dimensions. J. European Math. Soc. 16 (2014), 201-234.

[2] L. Ambrosio and G. Crippa, Existence, uniqueness, stability and differentiability properties of the flow associated to weakly differentiable vector fields. In: Transport equations and multi-D hyperbolic conservation laws, 3-57. Lecture Notes Un. Mat. Ital. 5, Springer, Berlin, 2008.

[3] F. Ancona and A. Bressan, Patchy vector fields and asymptotic stabilization, Control, Opt. Calc. Var. 4 (1999) 419-444.

[4] P. Binding, The differential equation $\dot{x}=f \circ x$. J. Differential Equations 31 (1979), 183-199.

[5] A. Bressan, Unique solutions for a class of discontinuous differential equations. Proc. Amer. Math. Soc. 104 (1988), 772-778.

[6] A. Bressan and G. Colombo, Existence and continuous dependence for discontinuous O.D.E's, Boll. Un. Mat. Italiana, B, 4 (1990) 295-311.

[7] A. Bressan, M. Mazzola, and K. T. Nguyen, Diffusion approximations of Markovian solutions to discontinuous ODEs, to appear.

[8] A. Bressan and W. Shen, Uniqueness for discontinuous O.D.E. and conservation laws. Nonlinear Anal. 34 (1998), 637-652.

[9] F. H. Clarke, Y. S. Ledyaev, R. J. Stern, and P. R. Wolenski, Nonsmooth Analysis and Control Theory. Springer, New York, 1998. 
[10] F. Colombini and J. Rauch, Uniqueness in the Cauchy problem for transport in $\mathbb{R}^{2}$ and $\mathbb{R}^{1+2}$. J. Differential Equations 211 (2005), 162-167.

[11] R. M. Colombo and A. Marson, A Hölder continuous ODE related to traffic flow. Proc. Roy. Soc. Edinburgh, Sect. A, 133 (2003), 759-772.

[12] C. Dafermos, Generalized characteristics and the structure of solutions of hyperbolic conservation laws. Indiana Univ. Math. J. 26 (1977), 1097-1119.

[13] C. Dafermos, Generalized characteristics in hyperbolic systems of conservation laws. Arch. Rational Mech. Anal. 107 (1989), 127-155.

[14] L. Dieci and L. Lopez, A survey of numerical methods for IVPs of ODEs with discontinuous right-hand side. J. Comput. Appl. Math. 236 (2012), 3967-3991.

[15] R. J. DiPerna and P. L. Lions, Ordinary differential equations, transport theory and Sobolev spaces. Invent. Math. 98, (1989), 511-547.

[16] W. E and E. Vanden-Eijnden, A note on generalized flows. Phys. D 183 (2003), 159174.

[17] A. Filippov, Differential Equations with Discontinuous Right-Hand Sides. Kluwer Academic, Dordrecht, 1988.

[18] F. Flandoli and J. Langa, Markov attractors: a probabilistic approach to multivalued flows. Stoch. Dyn. 8 (2008), 59-75.

[19] A. Marigo and B. Piccoli, Regular syntheses and solutions to discontinuous ODEs. ESAIM Control Optim. Calc. Var. 7 (2002), 291-307.

[20] R. Sentis, Équations différentielles à second membre mesurable. Boll. Un. Mat. Italiana, $B 15$ (1978), 724-742.

[21] B. Piccoli and H. Sussmann, Regular synthesis and sufficiency conditions for optimality. SIAM J. Control Optim. 39 (2000), 359-410. 